Electronic Supporting Information (ESI)

\title{
Soluble supertetrahedral chalcogenido T4 clusters: High stability and enhanced hydrogen evolution activities
}

Minting Hao, ${ }^{a}$ Qianqian Hu*, ${ }^{a}$ Yuanfei Zhang, ${ }^{a}$ Mingbu Luo, ${ }^{a}$ Yanqi Wang, ${ }^{a}$ Bing $\mathrm{Hu},{ }^{a}$ Jianrong Li* ${ }^{*}{ }^{a}$ and Xiaoying Huang ${ }^{a}$

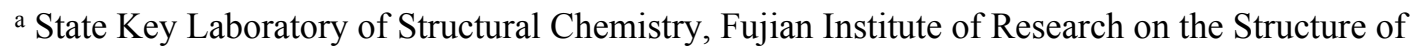
Matter, Chinese Academy of Sciences, Fuzhou, Fujian, 350002, P. R. China

Fax: +8659163173145; Tel: +8659163173145; E-mail: huqianqian@fjirsm.ac.cn

Fax: +86 591 63173145; Tel: +8659163173145; E-mail:jrli@fjirsm.ac.cn 


\section{Index}

\section{Characterizations and performance tests}

\section{Photocatalytic activity comparison}

(1) Table S1. Selected data for chalcogenide crystals applied in photocatalytic $\mathrm{H}_{2}$ production or dye degradation.

3. More crystal structural details

(1) Table S2. Crystal data and structure refinement details for the title compounds.

(2) Table S3. Atomic coordinates $\left(\times 10^{4}\right)$ and equivalent isotropic displacement parameters $\left(\mathrm{A}^{2} \times 10^{3}\right)$ for the atoms in anionic cluster of T4-1. $U(e q)$ is defined as one third of the trace of the orthogonalized $U i j$ tensor.

(3) Table S4. Atomic coordinates $\left(\times 10^{4}\right)$ and equivalent isotropic displacement parameters $\left(\mathrm{A}^{2} \times 10^{3}\right)$ for the atoms in anionic cluster of T4-2. $U(e q)$ is defined as one third of the trace of the orthogonalized Uij tensor and the refined site occupancy factors for $\mathrm{S} / \mathrm{Se}$ in T4-2 at various unique crystallographic sites.

(4) Table S5. Atomic coordinates $\left(\times 10^{4}\right)$ and equivalent isotropic displacement parameters $\left(\mathrm{A}^{2} \times 10^{3}\right)$ for the atoms in anionic cluster of T4-3. U(eq) is defined as one third of the trace of the orthogonalized Uij tensor.

(5) Table S6. Selected bond lengths $[\AA]$ and angles $\left[{ }^{\circ}\right]$ for T4-1.

(6) Table S7. Selected bond lengths $[\AA]$ and angles $\left[{ }^{\circ}\right]$ for T4-2.

(7) Table S8. Selected bond lengths $[\AA]$ and angles $\left[^{\circ}\right]$ for T4-3.

(8) Figure S1. (a) Arrangement way of anions in T4-1 and T4-3; (b) arrangement way of anions in T4-2.

\section{More characterizations}

(1) Figure S2. PXRD patterns of title compounds and that simulated from single crystal X-ray structures.

(2) Figure S3. (a) TGA curves of title compounds; (b)-(d) PXRD patterns of the TGA residues of T4-1, T4-2 and T4-3 compared with that of corresponding phases based on JCPDS cards.

(3) Figure S4. EDS spectra of T4-1 (a), T4-2 (b) and T4-3 (c). The contents of chemical elements in T4 clusters are $\mathrm{CdIn}_{4.79} \mathrm{~S}_{7.23} \mathrm{Cl}_{1.30}$ for T4-1, $\mathrm{CdIn}_{5.40} \mathrm{~S}_{4.72} \mathrm{Se}_{6.02} \mathrm{Cl}_{1.35}$ for T4-2 and $\mathrm{CdIn}_{5.03} \mathrm{Se}_{10.20} \mathrm{Cl}_{1.21}$ for T4-3, respectively.

(4) Table S9. The contents of cadmium and indium in T4 clusters.

(5) Figure S5. The measured mass spectrum (bottom) of the [(BMMim) $\left.\left(\mathrm{Cd}_{3} \operatorname{In}_{17} \mathrm{Se}_{31}\right)\right]^{4-}$ clusters is comparable with that simulated from isotopic patterns (top).

(6) Figure S6. The measured mass spectrum (bottom) of the $\left[\mathrm{H}_{2}\left(\mathrm{Cd}_{3} \operatorname{In}_{17} \mathrm{Se}_{31}\right)\right]^{4-}$ clusters is comparable with that simulated from isotopic patterns (top).

(7) Figure S7. The measured mass spectrum (bottom) of the $\left[(\mathrm{BMMim})_{2}\left(\mathrm{Cd}_{3} \mathrm{In}_{17} \mathrm{Se}_{31}\right)\right]^{3-}$ clusters is comparable with that simulated from isotopic patterns (top).

(8) Figure S8. (a) Positive mode ESI-MS spectrum of T4-2 solution; (b) negative mode ESI-MS spectrum of T4-2 solution; (c) positive mode ESI-MS spectrum of T4-3 solution; (d) negative mode ESI-MS spectrum of T4-3 solution kept in the ambient conditions for 7 days, $10 \mathrm{mg}$ samples were dispersed in $4 \mathrm{~mL}$ DMSO, which were diluted by methanol and immediately injected into the MS instrument.

(9) Figure S9. Photos of the dispersed T4-2 (a) and T4-3 (b) clusters in DMSO solution (20 mg/mL) under ambient condition for different times.

(10) Figure S10. Mott-schottky plots of the title compounds at different frequency.

(11) Table S10. Calculated results for the band positions of T4-1, T4-2 and T4-3.

(12) Figure S11. Photos of T4-3 solution before (a) and after (b) photocatalytic reaction without cocatalyst Pt; photos of T4-3 solution before (c) and after (d) photocatalytic reaction with cocatalyst Pt.

(13) Figure S12. Liquid state UV-Vis absorption spectra for T4-2 and T4-3 before and after photocatalytic reaction.

(14) Figure S13. FT-IR spectra of T4-1 (a), T4-2 (b), and T4-3 (c). 


\section{Characterization and performance tests}

X-ray Crystal Structure Determination: Single-crystal X-ray diffraction (SCXRD) data were collected on a Rigaku MM007 CCD diffractometer with nitrogen-flow temperature controller using graphite-monochromated MoKa radiation $(\lambda=0.71073 \AA)$. All structures were solved by direct methods and refined by full-matrix least squares techniques in SHELX2016 program package. ${ }^{1}$ The organic cations and lattice solvent molecules were highly disordered so that they could not be reliably determined solely via SCXRD. Therefore, the SQUEEZE routine of PLATON was applied. The formulas of title compounds were determined according to other characterizations such as Elemental analysis (EA), ICP, FT-IR, ESI-MS and NMR. The three Cd atoms and one In atom were dealt with to co-occupy four unique metal sites uniformly on the faces of the cluster with the SOFs of 0.75 for $\mathrm{Cd}$ and 0.25 for In for each site. For T4-2, firstly the $\mathrm{SOF}$ ratio of $\mathrm{S}: \mathrm{Se}$ for each $\mathrm{S} / \mathrm{Se}$ site was refined freely, leading to a whole $\mathrm{S} / \mathrm{Se}$ ratio close to that of the formula derived from other characterizations; finally SUMP was applied to restrict the refined whole $\mathrm{S} / \mathrm{Se}$ ratio to be the same as that displayed in formula.

Measurements and characterizations: Elemental analyses (EA) of C, H, N, S was performed on a German Elementary Vario EL III instrument. Energy-dispersive spectroscopy (EDS) was recorded on a JEOL JSM-6700F scanning electron microscope. Elemental contents of $\mathrm{Cd}$, In and Se were determined by inductively coupled plasma-atomic emission spectrometry (ICP-AES) by using an ultima 2 instrument. PXRD patterns were measured on a Rigaku Miniflex II diffractometer by using $\mathrm{Cu}-\mathrm{K} \alpha$ radiation $(\lambda=1.54178 \AA$ ). Thermogravimetric analysis (TGA) was carried out on a NETZSCH STA 449F3 unit at a heating rate of $5 \mathrm{~K} \mathrm{~min}^{-1}$ under a $\mathrm{N}_{2}$ atmosphere from 20 to $600{ }^{\circ} \mathrm{C}$. The solid state UV-Vis diffuse reflectance spectra were measured at room temperature with a Shimadzu UV-2600 UV-Vis spectrophotometer. $\mathrm{A} \mathrm{BaSO}_{4}$ plate was used as a standard ( $100 \%$ reflectance). The absorption spectra were calculated from reflectance spectra by using the Kubelka-Munk function $\alpha / S=(1-R)^{2} / 2 R$, where $\alpha$ is the absorption coefficient, $S$ is the scattering coefficient which is practically independent of wavelength when the particle size is larger than $5 \mu \mathrm{m}$ and $R$ is the reflection coefficient of the sample. ${ }^{2}$ The data of FT-IR were harvested on a PerkinElmer Spectrum 100 FT-IR Spectrometer from 400 to $4000 \mathrm{~cm}^{-1}$ (Figure S9). ${ }^{1} \mathrm{H}$ NMR spectra of T4-2 and T4-3 were recorded on a Bruker AVANCE III spectrometer with Deuterated DMSO as solvent. TEM and HRTEM images were acquired on Transmission Electron Microscopy (Tecnai G2 F20, FEI, USA). ${ }^{13} \mathrm{C}$ NMR spectra of T4-1 were recorded on a Bruker AVANCE III spectrometer. ESI-MS was carried out on an Impact II UHR-TOF instrument (Bruker) with $10 \mathrm{mg}$ samples dispersed in $4 \mathrm{~mL}$ DMSO after ultrasonic treatment in an ultrasonic cleaner. The light emission spectra have been conducted on FLS980 spectrograph.

Photocatalytic hydrogen generation: Typically, the progress of $\mathrm{H}_{2}$ production took place in a closed vacuum gas-circulation system (CEL-SPH2N-D9) using a top window. $25 \mathrm{mg}$ samples were firstly added into $0.5 \mathrm{~mL}$ DMSO for ultrasonic treatment of about $20 \mathrm{~min}$, and then mixed with $89.5 \mathrm{~mL} \mathrm{H}_{2} \mathrm{O}$ and $10 \mathrm{~mL}$ triethanolamine. The mixtures were poured into a $250 \mathrm{~mL}$ quartz reactor, to which $265 \mathrm{uL}$ (equal to $2 \mathrm{wt}$. \% Pt) $\mathrm{H}_{2} \mathrm{PtCl}_{4}$ was added (no $\mathrm{H}_{2}$ signal has been detected by gas chromatography without adding $\mathrm{H}_{2} \mathrm{PtCl}_{4}$ ). Next, this system was evacuated (Ar as carrier gas) and then irradiated by a $300 \mathrm{~W}$ Xe lamp with a cutoff filter $\left(\lambda \geq 420 \mathrm{~nm}\right.$ ). Data of $\mathrm{H}_{2}$ production were measured via gas chromatograph. To make sure the proton source is $\mathrm{H}_{2} \mathrm{O}$ rather 
than DMSO, we conducted photocatalytic experiment under the same conditions except that 89.5 $\mathrm{mL} \mathrm{H}_{2} \mathrm{O}$ was replaced by $89.5 \mathrm{~mL}$ DMSO. The result of negligible $\mathrm{H}_{2}$ production (not shown in the data) confirmed that $\mathrm{H}$ atoms of acquired $\mathrm{H}_{2}$ came from $\mathrm{H}_{2} \mathrm{O}$. Photocatalytic activities of the title compounds in solid state were tested in the same closed vacuum gas-circulation system. The reaction mixture was prepared as follows: $25 \mathrm{mg}$ samples were ground and mixed with $90 \mathrm{~mL}$ $\mathrm{H}_{2} \mathrm{O}, 10 \mathrm{~mL}$ TEOA after ultrasonic treatment. Then the mixtures were transferred to a $250 \mathrm{~mL}$ quartz reactor, to which $0.5 \mathrm{~mL}$ DMSO and $265 \mu \mathrm{L}$ (equal to 2 wt. \% Pt) $\mathrm{H}_{2} \mathrm{PtCl}_{4}$ were added in sequence.

Electrochemical measurements. a) Photocurrent density measurement. Photocurrent density measurement was conducted using a CHI660E Electrochemical Workstation using a 3 electrode system with Indium-Tin Oxide (ITO) glass working electrode, a platinum wire counter electrode, and $\mathrm{Ag} / \mathrm{AgCl}$ reference electrode. $\mathrm{Na}_{2} \mathrm{SO}_{4}$ aqueous solution $(0.1 \mathrm{M})$ was used as the supporting electrolyte solution. A working electrode was prepared according to the following process: $2 \mathrm{mg}$ samples are dissolved in $40 \mu \mathrm{L}$ DMSO after ultrasonication, and was then dip-coated on a $10 \times 10 \mathrm{~mm}$ area of a $10 \times 40 \mathrm{~mm}$ ITO glass electrode. Finally, this ITO glass working electrode was dried at $60{ }^{\circ} \mathrm{C}$ under vacuum for 3 hours. b) Mott-Schottky plots. Mott-Schottky plots were generated by conducting impedance- frequency measurement. The capacitance of the semiconductor-electrolyte interface was collected at different frequency from 500 to $3000 \mathrm{~Hz}$ in the same system for photocurrent density measurement. But the treatment of ITO glass working electrode is different. A working electrode was prepared according to the following process: $5 \mathrm{mg}$ compounds was dispersed in a mixed solution containing $420 \mu \mathrm{L}$ alcohol, $30 \mu \mathrm{L} \mathrm{H}_{2} \mathrm{O}$ and $50 \mu \mathrm{L}$ Nafion D-520 dispersion, which was then dip-coated on a $10 \times 10 \mathrm{~mm}$ area of a $10 \times 40 \mathrm{~mm}$ ITO glass electrode. 


\section{Photocatalytic activity comparison}

Table S1. Selected data for chalcogenide crystals applied in photocatalytic $\mathrm{H}_{2}$ production or dye degradation.

\begin{tabular}{|c|c|c|c|c|}
\hline Formula & Activity & $\begin{array}{l}\text { Light } \\
\text { source }\end{array}$ & $\begin{array}{c}\text { Sacrificial } \\
\text { agent }\end{array}$ & Ref. \\
\hline $\mathrm{Na}_{14} \mathrm{In}_{17} \mathrm{Cu}_{3} \mathrm{~S}_{35} \cdot x \mathrm{H}_{2} \mathrm{O}$ & $18 \mu \mathrm{mol} \cdot \mathrm{h}^{-1} \cdot \mathrm{g}^{-1}$ & Visible light & $\mathrm{Na}_{2} \mathrm{~S} / \mathrm{Na}_{2} \mathrm{SO}_{3}$ & 3 \\
\hline$\left[\mathrm{Cu}_{8} \mathrm{Ge}_{5} \mathrm{~S}_{16}\right] \cdot x$ (solvent) & $2.64 \mu \mathrm{mol} \cdot \mathrm{h}^{-1} \cdot \mathrm{g}^{-1}$ & Visible light & $\mathrm{Na}_{2} \mathrm{SO}_{3}$ & 4 \\
\hline$\left(\mathrm{Cu}_{7} \mathrm{In}_{28} \mathrm{~S}_{53}\right) \cdot\left(\mathrm{C}_{8} \mathrm{H}_{18} \mathrm{NH}_{2}\right)_{15}$ & $3.8 \mu \mathrm{mol} \cdot \mathrm{h}^{-1} \cdot \mathrm{g}^{-1}$ & Visible light & $\mathrm{Na}_{2} \mathrm{~S}$ & 5 \\
\hline$\left(\mathrm{Zn}_{0.81} \mathrm{Ge}_{3.19} \mathrm{~S}_{8}\right)^{1.62-}$ & $2000 \mu \mathrm{mol} \cdot \mathrm{h}^{-1} \cdot \mathrm{g}^{-1}$ & UV light & $\mathrm{Na}_{2} \mathrm{~S} / \mathrm{Na}_{2} \mathrm{SO}_{3}$ & 6 \\
\hline $\begin{array}{c}{\left[\mathrm{Mn}_{2} \mathrm{Ga}_{4} \mathrm{Sn}_{4} \mathrm{~S}_{20}\left[\mathrm{Mn}_{\left.2(\mathrm{en})_{5}\right]_{2}}\right.\right.} \\
\cdot 4 \mathrm{H}_{2} \mathrm{O}\end{array}$ & $\begin{array}{l}\text { Photodegradation: } \mathrm{MV}^{[\mathrm{a}]} \\
\text { Completion time: } 2 \mathrm{~h}\end{array}$ & Visible light & --- & 7 \\
\hline$(\mathrm{Bmmim})_{8} \mathrm{In}_{8} \mathrm{Sn}_{8} \mathrm{Se}_{30}\left(\mathrm{Se}_{4}\right)_{2}$ & $\begin{array}{l}\text { Photodegradation: } \mathrm{MO}^{[\mathrm{b}]} \\
\text { Completion time: } 15 \mathrm{~min}\end{array}$ & Visible light & --- & 8 \\
\hline$[\mathrm{Bmmim}]_{5}\left[\operatorname{In}_{10} \mathrm{~S}_{16} \mathrm{Cl}_{3}(\mathrm{Bim})\right]$ & $\begin{array}{l}\text { Photodegradation: } \mathrm{MO} \\
\text { Completion time: } 2 \mathrm{~h}\end{array}$ & UV light & --- & 9 \\
\hline
\end{tabular}

${ }^{[\mathrm{a}]}$ : methyl viologen; ${ }^{[\mathrm{b}]}$ : methyl orange

\section{More crystal structural details}

Table S2. Crystal data and structure refinement details for the title compounds.

\begin{tabular}{|c|c|c|c|}
\hline Compound reference & T4-1 & T4-2 & T4-3 \\
\hline Empirical formula & $\begin{array}{c}\mathrm{C}_{81} \mathrm{H}_{153} \mathrm{~N}_{18} \mathrm{Cl}_{4} \\
\mathrm{~S}_{31} \mathrm{Cd}_{3} \mathrm{In}_{17}\end{array}$ & $\begin{array}{c}\mathrm{C}_{81} \mathrm{H}_{153} \mathrm{~N}_{18} \mathrm{~S}_{13} \mathrm{Cl}_{4} \\
\mathrm{Se}_{18} \mathrm{Cd}_{3} \mathrm{In}_{17}\end{array}$ & $\begin{array}{c}\mathrm{C}_{91} \mathrm{H}_{161} \mathrm{~N}_{20} \mathrm{Cl}_{4} \\
\mathrm{Se}_{31} \mathrm{Cd}_{3} \mathrm{In}_{17}\end{array}$ \\
\hline Formula Mass & 4804.00 & 5648.21 & 6414.09 \\
\hline Crystal system & hexagonal & triclinic & hexagonal \\
\hline Space group & $P 6_{3} / m$ & $P-1$ & $P 6_{3} / m$ \\
\hline$a / \AA$ & $21.6032(5)$ & $20.095(4)$ & $21.923(4)$ \\
\hline$b / \AA$ & $21.6032(5)$ & $20.477(4)$ & $21.923(4)$ \\
\hline$c / \AA$ & $38.2733(14)$ & $23.679(4)$ & $43.837(11)$ \\
\hline$\alpha /^{\circ}$ & 90 & $66.161(10)$ & 90 \\
\hline$\beta /{ }^{\circ}$ & 90 & $71.126(12)$ & 90 \\
\hline$\gamma /{ }^{\circ}$ & 120 & $64.248(9)$ & 120 \\
\hline$V / \AA^{3}$ & $15469.0(9)$ & $7896(3)$ & $18247(8)$ \\
\hline$Z$ & 4 & 2 & 4 \\
\hline$\lambda / \AA$ & 0.71073 & 0.71073 & 0.71073 \\
\hline $\mathrm{T} / \mathrm{K}$ & $100(2)$ & $100(2)$ & $100(2)$ \\
\hline$F(000)$ & 9224 & 5260 & 11784 \\
\hline$\rho_{\text {calcu }} / \mathrm{g} \mathrm{cm}^{-3}$ & 2.063 & 2.376 & 2.335 \\
\hline$\mu / \mathrm{mm}-1$ & 3.409 & 7.250 & 8.723 \\
\hline No. of Reflection collected & 122632 & 86337 & 117971 \\
\hline No. of Reflection unique & 11977 & 35852 & 14083 \\
\hline No. of parameters & 166 & 527 & 166 \\
\hline$R_{\text {int }}$ & 0.0422 & 0.0665 & 0.0919 \\
\hline${ }^{[\mathrm{a}]} R_{1}$ value $(I>2 \sigma(I))$ & 0.0387 & 0.0676 & 0.0911 \\
\hline${ }^{[\mathrm{b}]} w R\left(F^{2}\right)$ value $(I>2 \sigma(I))$ & 0.0849 & 0.1804 & 0.1385 \\
\hline$R_{1}$ value (all data) & 0.0674 & 0.0964 & 0.1001 \\
\hline$w R\left(F^{2}\right)$ value (all data) & 0.0997 & 0.1951 & 0.1425 \\
\hline$G O F$ & 1.017 & 1.074 & 1.018 \\
\hline CCDC Number & 1820015 & 1820016 & 1820017 \\
\hline
\end{tabular}

[a] $R_{1}=\sum\left\|F_{\mathrm{o}}|-| F_{\mathrm{c}}\right\| / \sum\left|F_{\mathrm{o}}\right| .[\mathrm{b}] w R_{2}=\left[\sum w\left(F_{\mathrm{o}}^{2}-F_{\mathrm{c}}^{2}\right)^{2} / \sum w\left(F_{\mathrm{o}}^{2}\right)^{2}\right]^{1 / 2}$. 
Table S3. Atomic coordinates $\left(\times 10^{4}\right)$ and equivalent isotropic displacement parameters $\left(\mathrm{A}^{2} \times 10^{3}\right)$ for the atoms in anionic cluster of T4-1. $U(e q)$ is defined as one third of the trace of the orthogonalized $U i j$ tensor.

\begin{tabular}{ccccc|ccccc}
\hline Atom & $x$ & $y$ & $z$ & $U(e q)$ & Atom & $x$ & $y$ & $z$ & $U(e q)$ \\
\hline $\mathrm{M}(1)$ & $3801(1)$ & $5881(1)$ & $5512(1)$ & $91(1)$ & $\mathrm{S}(5)$ & $3023(1)$ & $4636(1)$ & $5728(1)$ & $92(1)$ \\
$\mathrm{M}(2)$ & 3333 & 6667 & $6389(1)$ & $90(1)$ & $\mathrm{S}(6)$ & 3333 & 6667 & $5731(1)$ & $85(1)$ \\
$\mathrm{In}(1)$ & 3333 & 6667 & $3898(1)$ & $110(1)$ & $\mathrm{S}(7)$ & $1025(1)$ & $3000(1)$ & $5711(1)$ & $119(1)$ \\
$\mathrm{In}(2)$ & $4580(1)$ & $7129(1)$ & $4661(1)$ & $93(1)$ & $\mathrm{S}(8)$ & $1280(1)$ & $4956(1)$ & $5724(1)$ & $92(1)$ \\
$\mathrm{In}(3)$ & $1786(1)$ & $4210(1)$ & $5508(1)$ & $94(1)$ & $\mathrm{S}(9)$ & $4248(1)$ & $5128(1)$ & $6586(1)$ & $105(1)$ \\
$\mathrm{In}(4)$ & $3036(1)$ & $4676(1)$ & $6378(1)$ & $94(1)$ & $\mathrm{S}(10)$ & $2553(1)$ & $5428(1)$ & $6613(1)$ & $93(1)$ \\
$\mathrm{In}(5)$ & $1325(1)$ & $4985(1)$ & $6378(1)$ & $94(1)$ & $\mathrm{S}(11)$ & $2294(1)$ & $3470(1)$ & $6587(1)$ & $118(1)$ \\
$\mathrm{In}(6)$ & $1125(1)$ & $3143(1)$ & $6344(1)$ & $114(1)$ & $\mathrm{S}(12)$ & $562(1)$ & $3782(1)$ & $6591(1)$ & $116(1)$ \\
$\mathrm{S}(2)$ & $4587(1)$ & $7106(1)$ & $4030(1)$ & $114(1)$ & $\mathrm{Cl}(1)$ & 3333 & 6667 & $3244(1)$ & $228(2)$ \\
$\mathrm{S}(3)$ & $5788(1)$ & $7589(1)$ & $4874(1)$ & $102(1)$ & $\mathrm{Cl}(2)$ & $370(2)$ & $1889(1)$ & $6554(1)$ & $226(1)$ \\
$\mathrm{S}(4)$ & $3803(1)$ & $5867(1)$ & $4859(1)$ & $92(1)$ & & & & & \\
\hline
\end{tabular}

*: $\mathrm{M}(1)=0.75 \mathrm{Cd}(1)+0.25 \operatorname{In}(7) ; \mathrm{M}(2)=0.75 \mathrm{Cd}(2)+0.25 \operatorname{In}(8)$

Table S4. Atomic coordinates $\left(\times 10^{4}\right)$ and equivalent isotropic displacement parameters $\left(\mathrm{A}^{2} \times 10^{3}\right)$ for the atoms in anionic cluster of T4-2. $U(e q)$ is defined as one third of the trace of the orthogonalized Uij tensor and the refined site occupancy factors for $\mathrm{S} / \mathrm{Se}$ in T4-2 at various unique crystallographic sites.

\begin{tabular}{cccccc}
\hline Atom & $x$ & $y$ & $z$ & $U(e q)$ & SOF $(\mathrm{Se} / \mathrm{S})$ \\
\hline $\mathrm{M}(1)$ & $5884(1)$ & $5346(1)$ & $7986(1)$ & $47(1)$ & \\
$\mathrm{M}(2)$ & $5614(1)$ & $5725(1)$ & $6196(1)$ & $48(1)$ & \\
$\mathrm{M}(3)$ & $7686(1)$ & $5282(1)$ & $6517(1)$ & $48(1)$ & \\
$\mathrm{M}(4)$ & $5981(1)$ & $7344(1)$ & $6570(1)$ & $48(1)$ & \\
$\mathrm{In}(1)$ & $2313(1)$ & $7757(1)$ & $7662(1)$ & $66(1)$ & \\
$\mathrm{In}(2)$ & $7156(1)$ & $1896(1)$ & $7514(1)$ & $68(1)$ & \\
$\mathrm{In}(3)$ & $8257(1)$ & $6452(1)$ & $8574(1)$ & $64(1)$ & \\
$\mathrm{In}(4)$ & $7479(1)$ & $7568(1)$ & $3498(1)$ & $62(1)$ & \\
$\mathrm{In}(5)$ & $3825(1)$ & $5787(1)$ & $7665(1)$ & $50(1)$ & \\
$\mathrm{In}(6)$ & $4198(1)$ & $7402(1)$ & $8036(1)$ & $50(1)$ & \\
$\mathrm{In}(7)$ & $3927(1)$ & $7780(1)$ & $6239(1)$ & $50(1)$ & \\
$\mathrm{In}(8)$ & $5495(1)$ & $3761(1)$ & $7607(1)$ & $51(1)$ & \\
$\mathrm{In}(9)$ & $5715(1)$ & $7723(1)$ & $4801(1)$ & $48(1)$ & \\
$\mathrm{In}(10)$ & $6249(1)$ & $6957(1)$ & $8347(1)$ & $52(1)$ & \\
$\mathrm{In}(11)$ & $7428(1)$ & $5653(1)$ & $4749(1)$ & $50(1)$ & \\
$\mathrm{In}(12)$ & $7322(1)$ & $3691(1)$ & $6140(1)$ & $54(1)$ & \\
$\mathrm{In}(13)$ & $7805(1)$ & $7268(1)$ & $5126(1)$ & $51(1)$ & \\
$\mathrm{In}(14)$ & $7584(1)$ & $3298(1)$ & $7931(1)$ & $53(1)$ & \\
$\mathrm{In}(15)$ & $8063(1)$ & $6884(1)$ & $6878(1)$ & $53(1)$ & \\
$\mathrm{In}(16)$ & $7954(1)$ & $4878(1)$ & $8292(1)$ & $51(1)$ & \\
$\mathrm{Q}(1)$ & $2530(1)$ & $8451(1)$ & $6502(1)$ & $70(1)$ & $0.654(3) / 0.346(3)$ \\
$\mathrm{Q}(2)$ & $2807(1)$ & $8031(1)$ & $8370(1)$ & $68(1)$ & $0.745(3) / 0.255(3)$ \\
$\mathrm{Q}(3)$ & $2426(1)$ & $6387(1)$ & $7947(1)$ & $64(1)$ & $0.659(3) / 0.341(3)$
\end{tabular}




\begin{tabular}{|c|c|c|c|c|c|}
\hline $\mathrm{Q}(4)$ & 4194(1) & $6361(1)$ & $6473(1)$ & $45(1)$ & $0.361(3) / 0.639(3)$ \\
\hline $\mathrm{Q}(5)$ & $4102(1)$ & $4373(1)$ & $7956(1)$ & $57(1)$ & $0.778(3) / 0.222(3)$ \\
\hline $\mathrm{Q}(6)$ & $4471(1)$ & $5961(1)$ & $8350(1)$ & $48(1)$ & $0.496(3) / 0.504(3)$ \\
\hline $\mathrm{Q}(7)$ & $4577(1)$ & $8024(1)$ & $6858(1)$ & $50(1)$ & $0.422(3) / 0.578(3)$ \\
\hline $\mathrm{Q}(8)$ & $4860(1)$ & $7657(1)$ & $8628(1)$ & $53(1)$ & $0.706(3) / 0.294(3)$ \\
\hline $\mathrm{Q}(9)$ & $4319(1)$ & $8402(1)$ & $5082(1)$ & $51(1)$ & $0.713(3) / 0.287(3)$ \\
\hline $\mathrm{Q}(10)$ & $5749(1)$ & $2364(1)$ & $7846(1)$ & $73(1)$ & $0.713(3) / 0.287(3)$ \\
\hline $\mathrm{Q}(11)$ & $6179(1)$ & $3925(1)$ & $8250(1)$ & $49(1)$ & $0.345(3) / 0.655(3)$ \\
\hline $\mathrm{Q}(12)$ & $5886(1)$ & $4309(1)$ & $6415(1)$ & $49(1)$ & $0.461(3) / 0.539(3)$ \\
\hline $\mathrm{Q}(13)$ & $6081(1)$ & $8307(1)$ & $3636(1)$ & $59(1)$ & $0.663(3) / 0.337(3)$ \\
\hline $\mathrm{Q}(14)$ & $6000(1)$ & $6298(1)$ & $5018(1)$ & $47(1)$ & $0.453(3) / 0.547(3)$ \\
\hline $\mathrm{Q}(15)$ & $6393(1)$ & $7974(1)$ & $5403(1)$ & $47(1)$ & $0.441(3) / 0.559(3)$ \\
\hline $\mathrm{Q}(16)$ & $6659(1)$ & $7576(1)$ & $7177(1)$ & $51(1)$ & $0.394(3) / 0.606(3)$ \\
\hline $\mathrm{Q}(17)$ & $6903(1)$ & $7154(1)$ & $8987(1)$ & $67(1)$ & $0.720(3) / 0.280(3)$ \\
\hline $\mathrm{Q}(18)$ & $6541(1)$ & $5510(1)$ & $8666(1)$ & $50(1)$ & $0.543(3) / 0.457(3)$ \\
\hline $\mathrm{Q}(19)$ & $8174(1)$ & $5823(1)$ & $5352(1)$ & $51(1)$ & $0.489(3) / 0.511(3)$ \\
\hline $\mathrm{Q}(20)$ & $7869(1)$ & $6179(1)$ & $3589(1)$ & $60(1)$ & $0.666(3) / 0.334(3)$ \\
\hline $\mathrm{Q}(21)$ & $7731(1)$ & $4244(1)$ & $4975(1)$ & $60(1)$ & $0.761(3) / 0.239(3)$ \\
\hline $\mathrm{Q}(22)$ & $7685(1)$ & $2275(1)$ & $6352(1)$ & $72(1)$ & $0.640(3) / 0.360(3)$ \\
\hline $\mathrm{Q}(23)$ & $8053(1)$ & $3847(1)$ & $6755(1)$ & $52(1)$ & $0.396(3) / 0.604(3)$ \\
\hline $\mathrm{Q}(24)$ & $8496(1)$ & $7477(1)$ & $5724(1)$ & $60(1)$ & $0.721(3) / 0.279(3)$ \\
\hline $\mathrm{Q}(25)$ & $8241(1)$ & $7852(1)$ & $3975(1)$ & $62(1)$ & $0.677(3) / 0.323(3)$ \\
\hline $\mathrm{Q}(26)$ & $7903(1)$ & 1881(1) & $8206(1)$ & $72(1)$ & $0.742(3) / 0.258(3)$ \\
\hline $\mathrm{Q}(27)$ & $8258(1)$ & $3460(1)$ & $8570(1)$ & $62(1)$ & $0.794(3) / 0.206(3)$ \\
\hline $\mathrm{Q}(28)$ & $8420(1)$ & $5447(1)$ & $7121(1)$ & $51(1)$ & $0.435(3) / 0.565(3)$ \\
\hline $\mathrm{Q}(29)$ & $8701(1)$ & $5017(1)$ & $8885(1)$ & $59(1)$ & $0.557(3) / 0.443(3)$ \\
\hline $\mathrm{Q}(30)$ & $8744(1)$ & $7129(1)$ & $7453(1)$ & $66(1)$ & $0.659(3) / 0.341(3)$ \\
\hline $\mathrm{Q}(31)$ & $6289(1)$ & $5929(1)$ & $6819(1)$ & $44(1)$ & $0.199(3) / 0.801(3)$ \\
\hline $\mathrm{Cl}(1)$ & $947(2)$ & $8388(2)$ & $7937(2)$ & $116(1)$ & \\
\hline $\mathrm{Cl}(2)$ & $7419(2)$ & $536(2)$ & $7727(2)$ & $108(1)$ & \\
\hline $\mathrm{Cl}(3)$ & $8950(2)$ & $6592(2)$ & $9195(2)$ & $95(1)$ & \\
\hline $\mathrm{Cl}(4)$ & $7894(2)$ & $8150(2)$ & $2377(1)$ & $110(1)$ & \\
\hline
\end{tabular}

*: $\mathrm{M}(1)=0.75 \mathrm{Cd}(1)+0.25 \mathrm{In}(1 \mathrm{~B}) ; \mathrm{M}(2)=0.75 \mathrm{Cd}(2)+0.25 \mathrm{In}(2 \mathrm{~B}) ; \mathrm{M}(3)=0.75 \mathrm{Cd}(3)+0.25 \operatorname{In}(3 \mathrm{~B}) ; \mathrm{M}(4)=$ $0.75 \mathrm{Cd}(4)+0.25 \operatorname{In}(4 \mathrm{~B})$

Table S5. Atomic coordinates $\left(\times 10^{4}\right)$ and equivalent isotropic displacement parameters $\left(\mathrm{A}^{2} \times 10^{3}\right)$ for the atoms in anionic cluster of T4-3. $U(e q)$ is defined as one third of the trace of the orthogonalized $U i j$ tensor.

\begin{tabular}{ccccc|ccccc}
\hline Atom & $x$ & $y$ & $z$ & $U(e q)$ & Atom & $x$ & $y$ & $z$ & $U(e q)$ \\
\hline $\mathrm{M}(1)$ & $3780(1)$ & $5837(1)$ & $5432(1)$ & $52(1)$ & $\mathrm{Se}(5)$ & $2953(1)$ & $4561(1)$ & $5624(1)$ & $51(1)$ \\
$\mathrm{M}(2)$ & 3333 & 6667 & $6231(1)$ & $57(1)$ & $\mathrm{Se}(6)$ & 3333 & 6667 & $5634(1)$ & $49(1)$ \\
$\operatorname{In}(1)$ & 3333 & 6667 & $3964(1)$ & $55(1)$ & $\mathrm{Se}(7)$ & $848(1)$ & $2907(1)$ & $5601(1)$ & $66(1)$ \\
$\operatorname{In}(2)$ & $4602(1)$ & $7116(1)$ & $4660(1)$ & $50(1)$ & $\mathrm{Se}(8)$ & $1173(1)$ & $4942(1)$ & $5616(1)$ & $54(1)$ \\
$\operatorname{In}(3)$ & $1687(1)$ & $4158(1)$ & $5422(1)$ & $52(1)$ & $\mathrm{Se}(9)$ & $4211(1)$ & $5008(1)$ & $6400(1)$ & $64(1)$ \\
$\operatorname{In}(4)$ & $2960(1)$ & $4597(1)$ & $6218(1)$ & $55(1)$ & $\operatorname{Se}(10)$ & $2511(1)$ & $5408(1)$ & $6447(1)$ & $56(1)$
\end{tabular}




\begin{tabular}{ccccc|ccccc}
$\operatorname{In}(5)$ & $1264(1)$ & $4998(1)$ & $6214(1)$ & $55(1)$ & $\mathrm{Se}(11)$ & $2170(1)$ & $3343(1)$ & $6407(1)$ & $67(1)$ \\
$\operatorname{In}(6)$ & $990(1)$ & $3093(1)$ & $6177(1)$ & $63(1)$ & $\operatorname{Se}(12)$ & $459(1)$ & $3777(1)$ & $6429(1)$ & $66(1)$ \\
$\operatorname{Se}(2)$ & $4621(1)$ & $7052(1)$ & $4081(1)$ & $58(1)$ & $\mathrm{Cl}(1)$ & 3333 & 6667 & $3400(1)$ & $90(2)$ \\
$\operatorname{Se}(3)$ & $5866(1)$ & $7618(1)$ & $4844(1)$ & $53(1)$ & $\mathrm{Cl}(2)$ & $177(2)$ & $1887(2)$ & $6365(1)$ & $96(1)$ \\
$\operatorname{Se}(4)$ & $3804(1)$ & $5811(1)$ & $4839(1)$ & $50(1)$ & & & & & \\
\hline
\end{tabular}

Table S6. Selected bond lengths $[\AA]$ and angles $\left[^{\circ}\right]$ for T4-1.

\begin{tabular}{|c|c|c|c|}
\hline $\mathrm{M}(1)-\mathrm{S}(4)$ & $2.4964(15)$ & $\operatorname{In}(3)-S(3) \# 2$ & $2.4268(16)$ \\
\hline $\mathrm{M}(1)-\mathrm{S}(5)$ & $2.4948(15)$ & $\operatorname{In}(3)-S(5)$ & $2.4968(16)$ \\
\hline $\mathrm{M}(1)-\mathrm{S}(6)$ & $2.5151(9)$ & $\operatorname{In}(3)-S(7)$ & $2.4163(17)$ \\
\hline $\mathrm{M}(1)-\mathrm{S}(8) \# 1$ & $2.4947(15)$ & $\operatorname{In}(3)-S(8)$ & $2.4973(16)$ \\
\hline $\mathrm{M}(2)-\mathrm{S}(6)$ & $2.518(3)$ & $\operatorname{In}(4)-S(5)$ & $2.4917(16)$ \\
\hline $\mathrm{M}(2)-\mathrm{S}(10) \# 1$ & $2.4963(15)$ & $\operatorname{In}(4)-S(9)$ & $2.4248(17)$ \\
\hline $\mathrm{M}(2)-\mathrm{S}(10) \# 2$ & $2.4963(15)$ & $\operatorname{In}(4)-S(10)$ & $2.4951(15)$ \\
\hline $\mathrm{M}(2)-\mathrm{S}(10)$ & $2.4963(15)$ & $\operatorname{In}(4)-S(11)$ & $2.4123(17)$ \\
\hline $\operatorname{In}(1)-S(2) \# 1$ & $2.4339(18)$ & $\operatorname{In}(5)-S(8)$ & $2.5044(16)$ \\
\hline $\operatorname{In}(1)-S(2) \# 2$ & $2.4338(18)$ & $\operatorname{In}(5)-S(9) \# 2$ & $2.4285(17)$ \\
\hline $\operatorname{In}(1)-S(2)$ & $2.4338(18)$ & $\operatorname{In}(5)-S(10)$ & $2.4968(15)$ \\
\hline $\operatorname{In}(1)-\mathrm{Cl}(1)$ & $2.504(5)$ & $\operatorname{In}(5)-S(12)$ & $2.4183(17)$ \\
\hline $\operatorname{In}(2)-S(2)$ & $2.4150(17)$ & $\operatorname{In}(6)-S(12)$ & $2.4393(19)$ \\
\hline $\operatorname{In}(2)-S(3)$ & $2.4220(16)$ & $\operatorname{In}(6)-\mathrm{Cl}(2)$ & $2.496(3)$ \\
\hline $\operatorname{In}(2)-S(4) \# 1$ & $2.4972(15)$ & $\operatorname{In}(6)-S(7)$ & $2.4414(19)$ \\
\hline $\operatorname{In}(2)-S(4)$ & $2.4994(15)$ & $\operatorname{In}(6)-S(11)$ & $2.4397(19)$ \\
\hline $\mathrm{S}(4)-\mathrm{M}(1)-\mathrm{S}(6)$ & $110.24(6)$ & $S(3) \# 2-\operatorname{In}(3)-S(5)$ & $108.99(6)$ \\
\hline $\mathrm{S}(5)-\mathrm{M}(1)-\mathrm{S}(4)$ & $108.87(5)$ & $\mathrm{S}(3) \# 2-\operatorname{In}(3)-\mathrm{S}(8)$ & $109.86(6)$ \\
\hline $\mathrm{S}(5)-\mathrm{M}(1)-\mathrm{S}(6)$ & $109.60(5)$ & $\mathrm{S}(5)-\operatorname{In}(3)-\mathrm{S}(8)$ & $112.58(5)$ \\
\hline $\mathrm{S}(8) \# 1-\mathrm{M}(1)-\mathrm{S}(4)$ & $108.81(5)$ & $S(7)-\operatorname{In}(3)-S(3) \# 2$ & $108.94(6)$ \\
\hline $\mathrm{S}(8) \# 1-\mathrm{M}(1)-\mathrm{S}(5)$ & $108.77(5)$ & $S(7)-\operatorname{In}(3)-S(5)$ & $108.33(6)$ \\
\hline $\mathrm{S}(8) \# 1-\mathrm{M}(1)-\mathrm{S}(6)$ & $110.52(5)$ & $S(7)-\operatorname{In}(3)-S(8)$ & $108.08(6)$ \\
\hline $\mathrm{S}(10) \# 1-\mathrm{M}(2)-\mathrm{S}(6)$ & $110.11(4)$ & $S(5)-\operatorname{In}(4)-S(10)$ & $112.44(5)$ \\
\hline $\mathrm{S}(10) \# 2-\mathrm{M}(2)-\mathrm{S}(6)$ & $110.11(4)$ & $\mathrm{S}(9)-\operatorname{In}(4)-\mathrm{S}(5)$ & $109.46(6)$ \\
\hline $\mathrm{S}(10)-\mathrm{M}(2)-\mathrm{S}(6)$ & $110.10(4)$ & $\mathrm{S}(9)-\operatorname{In}(4)-\mathrm{S}(10)$ & $109.76(6)$ \\
\hline $\mathrm{S}(10) \# 1-\mathrm{M}(2)-\mathrm{S}(10)$ & $108.83(4)$ & $S(11)-\operatorname{In}(4)-S(5)$ & $107.61(6)$ \\
\hline $\mathrm{S}(10) \# 2-\mathrm{M}(2)-\mathrm{S}(10)$ & $108.83(4)$ & $S(11)-\operatorname{In}(4)-S(9)$ & $109.12(6)$ \\
\hline $\mathrm{S}(10) \# 2-\mathrm{M}(2)-\mathrm{S}(10) \# 1$ & $108.83(4)$ & $S(11)-\operatorname{In}(4)-S(10)$ & $108.37(6)$ \\
\hline $\mathrm{S}(2)-\operatorname{In}(1)-\mathrm{S}(2) \# 1$ & $115.81(3)$ & $\mathrm{S}(9) \# 2-\operatorname{In}(5)-\mathrm{S}(8)$ & $108.78(6)$ \\
\hline $\mathrm{S}(2) \# 2-\operatorname{In}(1)-\mathrm{S}(2)$ & $115.81(3)$ & $\mathrm{S}(9) \# 2-\operatorname{In}(5)-\mathrm{S}(10)$ & $109.11(6)$ \\
\hline $\mathrm{S}(2) \# 2-\operatorname{In}(1)-\mathrm{S}(2) \# 1$ & $115.81(3)$ & $\mathrm{S}(10)-\operatorname{In}(5)-\mathrm{S}(8)$ & $113.01(5)$ \\
\hline $\mathrm{S}(2) \# 2-\operatorname{In}(1)-\mathrm{Cl}(1)$ & $101.98(4)$ & $S(12)-\operatorname{In}(5)-S(8)$ & $108.23(6)$ \\
\hline $\mathrm{S}(2) \# 1-\operatorname{In}(1)-\mathrm{Cl}(1)$ & $101.98(4)$ & $S(12)-\operatorname{In}(5)-S(9) \# 2$ & $109.46(6)$ \\
\hline $\mathrm{S}(2)-\operatorname{In}(1)-\mathrm{Cl}(1)$ & $101.98(4)$ & $S(12)-\operatorname{In}(5)-S(10)$ & $108.20(6)$ \\
\hline$S(2)-\operatorname{In}(2)-S(3)$ & $109.18(6)$ & $\mathrm{S}(7)-\operatorname{In}(6)-\mathrm{Cl}(2)$ & $102.37(9)$ \\
\hline
\end{tabular}




\begin{tabular}{cccc}
$\mathrm{S}(2)-\operatorname{In}(2)-\mathrm{S}(4) \# 1$ & $109.09(6)$ & $\mathrm{S}(11)-\operatorname{In}(6)-\mathrm{S}(7)$ & $115.33(7)$ \\
$\mathrm{S}(2)-\operatorname{In}(2)-\mathrm{S}(4)$ & $106.83(6)$ & $\mathrm{S}(11)-\operatorname{In}(6)-\mathrm{Cl}(2)$ & $102.23(9)$ \\
$\mathrm{S}(3)-\operatorname{In}(2)-\mathrm{S}(4) \# 1$ & $108.90(5)$ & $\mathrm{S}(12)-\operatorname{In}(6)-\mathrm{S}(7)$ & $114.71(7)$ \\
$\mathrm{S}(3)-\operatorname{In}(2)-\mathrm{S}(4)$ & $110.14(5)$ & $\mathrm{S}(12)-\operatorname{In}(6)-\mathrm{S}(11)$ & $115.22(7)$ \\
$\mathrm{S}(4) \# 1-\operatorname{In}(2)-\mathrm{S}(4)$ & $112.63(7)$ & $\mathrm{S}(12)-\operatorname{In}(6)-\mathrm{Cl}(2)$ & $104.43(9)$ \\
\hline
\end{tabular}

*: $\mathrm{M}(1)=0.75 \mathrm{Cd}(1)+0.25 \operatorname{In}(7) ; \mathrm{M}(2)=0.75 \mathrm{Cd}(2)+0.25 \operatorname{In}(8)$

Symmetry transformations used to generate equivalent atoms:

$\# 11-y, 1+x-y,+z ; \# 2+y-x, 1-x,+z$

Table S7. Selected bond lengths $[\AA]$ and angles $\left[{ }^{\circ}\right]$ for T4-2.

\begin{tabular}{|c|c|c|c|}
\hline $\mathrm{M}(1)-\mathrm{Q}(31)$ & $2.5406(18)$ & $\operatorname{In}(7)-Q(1)$ & $2.5230(14)$ \\
\hline$M(1)-Q(11)$ & $2.5458(16)$ & $\operatorname{In}(7)-Q(9)$ & $2.5306(13)$ \\
\hline $\mathrm{M}(1)-\mathrm{Q}(6)$ & $2.5644(14)$ & $\operatorname{In}(7)-Q(7)$ & $2.5506(15)$ \\
\hline $\mathrm{M}(1)-\mathrm{Q}(18)$ & $2.5773(14)$ & $\operatorname{In}(7)-Q(4)$ & $2.5638(16)$ \\
\hline$M(2)-Q(31)$ & $2.5434(17)$ & $\operatorname{In}(8)-Q(10)$ & $2.5242(15)$ \\
\hline $\mathrm{M}(2)-\mathrm{Q}(14)$ & $2.5514(15)$ & $\operatorname{In}(8)-Q(11)$ & $2.5492(16)$ \\
\hline $\mathrm{M}(2)-\mathrm{Q}(4)$ & $2.5536(15)$ & $\operatorname{In}(8)-Q(12)$ & $2.5709(16)$ \\
\hline $\mathrm{M}(2)-\mathrm{Q}(12)$ & $2.5635(15)$ & $\operatorname{In}(9)-Q(13)$ & $2.5213(14)$ \\
\hline $\mathrm{M}(3)-\mathrm{Q}(31)$ & $2.5257(18)$ & $\operatorname{In}(9)-Q(9)$ & $2.5290(13)$ \\
\hline $\mathrm{M}(3)-\mathrm{Q}(28)$ & $2.5536(15)$ & $\operatorname{In}(9)-Q(15)$ & $2.5718(15)$ \\
\hline$M(3)-Q(19)$ & $2.5547(15)$ & $\operatorname{In}(9)-Q(14)$ & $2.5745(15)$ \\
\hline$M(3)-Q(23)$ & $2.5581(16)$ & $\operatorname{In}(10)-Q(8)$ & $2.5261(13)$ \\
\hline$M(4)-Q(31)$ & $2.5332(18)$ & $\operatorname{In}(10)-Q(17)$ & $2.5368(13)$ \\
\hline$M(4)-Q(7)$ & $2.5445(15)$ & $\operatorname{In}(10)-Q(16)$ & $2.5611(17)$ \\
\hline$M(4)-Q(16)$ & $2.5566(16)$ & $\operatorname{In}(10)-Q(18)$ & $2.5793(15)$ \\
\hline$M(4)-Q(15)$ & $2.5595(15)$ & $\operatorname{In}(11)-Q(20)$ & $2.5202(14)$ \\
\hline $\operatorname{In}(1)-\mathrm{Cl}(1)$ & $2.463(3)$ & $\operatorname{In}(11)-Q(21)$ & $2.5328(14)$ \\
\hline $\operatorname{In}(1)-Q(1)$ & $2.5228(16)$ & $\operatorname{In}(11)-Q(14)$ & $2.5664(15)$ \\
\hline $\operatorname{In}(1)-Q(3)$ & $2.5280(16)$ & $\operatorname{In}(11)-Q(19)$ & $2.5784(15)$ \\
\hline $\operatorname{In}(1)-Q(2)$ & $2.5345(16)$ & $\operatorname{In}(12)-Q(21)$ & $2.5271(14)$ \\
\hline $\operatorname{In}(2)-\mathrm{Cl}(2)$ & $2.458(3)$ & $\operatorname{In}(12)-Q(22)$ & $2.5326(15)$ \\
\hline $\operatorname{In}(2)-Q(10)$ & $2.5195(17)$ & $\operatorname{In}(12)-Q(23)$ & $2.5601(16)$ \\
\hline $\operatorname{In}(2)-Q(22)$ & $2.5314(18)$ & $\operatorname{In}(12)-Q(12)$ & $2.5741(15)$ \\
\hline $\operatorname{In}(2)-Q(26)$ & $2.5400(15)$ & $\operatorname{In}(13)-Q(25)$ & $2.5210(14)$ \\
\hline $\operatorname{In}(3)-\mathrm{Cl}(3)$ & $2.489(3)$ & $\operatorname{In}(13)-Q(24)$ & $2.5306(14)$ \\
\hline $\operatorname{In}(3)-Q(29)$ & $2.5247(16)$ & $\operatorname{In}(13)-Q(15)$ & $2.5629(15)$ \\
\hline $\operatorname{In}(3)-Q(17)$ & $2.5378(16)$ & $\operatorname{In}(13)-Q(19)$ & $2.5800(15)$ \\
\hline $\operatorname{In}(3)-Q(30)$ & $2.5381(16)$ & $\operatorname{In}(14)-Q(27)$ & $2.5230(13)$ \\
\hline $\operatorname{In}(4)-\mathrm{Cl}(4)$ & $2.458(3)$ & $\operatorname{In}(14)-Q(26)$ & $2.5255(14)$ \\
\hline $\operatorname{In}(4)-Q(25)$ & $2.5262(16)$ & $\operatorname{In}(14)-Q(11)$ & $2.5380(17)$ \\
\hline $\operatorname{In}(4)-Q(13)$ & $2.5297(15)$ & $\operatorname{In}(14)-Q(23)$ & $2.5685(17)$ \\
\hline $\operatorname{In}(4)-Q(20)$ & $2.5351(15)$ & $\operatorname{In}(15)-Q(30)$ & $2.5164(14)$ \\
\hline $\operatorname{In}(5)-Q(3)$ & $2.5111(14)$ & $\operatorname{In}(15)-Q(24)$ & $2.5287(14)$ \\
\hline
\end{tabular}




\begin{tabular}{|c|c|c|c|}
\hline $\operatorname{In}(5)-Q(5)$ & $2.5303(14)$ & $\operatorname{In}(15)-Q(16)$ & $2.5511(16)$ \\
\hline $\operatorname{In}(5)-Q(4)$ & $2.5725(16)$ & $\operatorname{In}(15)-Q(28)$ & $2.5633(16)$ \\
\hline $\operatorname{In}(5)-Q(6)$ & $2.5828(14)$ & $\operatorname{In}(16)-Q(29)$ & $2.5216(14)$ \\
\hline $\operatorname{In}(6)-Q(2)$ & $2.5222(14)$ & $\operatorname{In}(16)-Q(27)$ & $2.5318(14)$ \\
\hline $\operatorname{In}(6)-Q(8)$ & $2.5312(13)$ & $\operatorname{In}(16)-Q(28)$ & $2.5644(16)$ \\
\hline $\operatorname{In}(6)-Q(7)$ & $2.5650(16)$ & $\operatorname{In}(16)-Q(18)$ & $2.5735(15)$ \\
\hline $\operatorname{In}(6)-Q(6)$ & $2.5782(15)$ & & \\
\hline $\mathrm{Q}(31)-\mathrm{M}(1)-\mathrm{Q}(11)$ & $110.38(6)$ & $\mathrm{Cl}(2)-\operatorname{In}(2)-(10)$ & $100.68(10)$ \\
\hline $\mathrm{Q}(31)-\mathrm{M}(1)-\mathrm{Q}(6)$ & $112.01(5)$ & $\mathrm{Cl}(2)-\operatorname{In}(2)-\mathrm{Q}(10)$ & $100.68(10)$ \\
\hline $\mathrm{Q}(11)-\mathrm{M}(1)-\mathrm{Q}(6)$ & $107.84(5)$ & $\mathrm{Cl}(2)-\operatorname{In}(2)-(22)$ & $100.52(10)$ \\
\hline $\mathrm{Q}(31)-\mathrm{M}(1)-\mathrm{Q}(18)$ & $112.66(5)$ & $\mathrm{Cl}(2)-\operatorname{In}(2)-\mathrm{Q}(22)$ & $100.52(10)$ \\
\hline $\mathrm{Q}(11)-\mathrm{M}(1)-\mathrm{Q}(18)$ & $107.78(5)$ & $\mathrm{Q}(10)-\operatorname{In}(2)-\mathrm{Q}(22)$ & $117.74(6)$ \\
\hline $\mathrm{Q}(6)-\mathrm{M}(1)-\mathrm{Q}(18)$ & $105.91(5)$ & $\mathrm{Cl}(2)-\operatorname{In}(2)-(26)$ & $103.38(9)$ \\
\hline $\mathrm{Q}(31)-\mathrm{M}(2)-\mathrm{Q}(14)$ & $111.37(5)$ & $\mathrm{Q}(25)-\operatorname{In}(13)-\mathrm{Q}(24)$ & $106.90(5)$ \\
\hline $\mathrm{Q}(31)-\mathrm{M}(2)-\mathrm{Q}(4)$ & $110.76(6)$ & $\mathrm{Q}(25)-\operatorname{In}(13)-\mathrm{Q}(15)$ & $108.57(5)$ \\
\hline $\mathrm{Q}(14)-\mathrm{M}(2)-\mathrm{Q}(4)$ & $107.39(5)$ & $\mathrm{Q}(24)-\operatorname{In}(13)-\mathrm{Q}(15)$ & $108.97(5)$ \\
\hline $\mathrm{Q}(31)-\mathrm{M}(2)-\mathrm{Q}(12)$ & $112.25(5)$ & $\mathrm{Q}(25)-\operatorname{In}(13)-\mathrm{Q}(19)$ & $108.59(5)$ \\
\hline $\mathrm{Q}(14)-\mathrm{M}(2)-\mathrm{Q}(12)$ & $107.54(5)$ & $\mathrm{Q}(24)-\operatorname{In}(13)-\mathrm{Q}(19)$ & $109.19(5)$ \\
\hline $\mathrm{Q}(4)-\mathrm{M}(2)-\mathrm{Q}(12)$ & $107.30(5)$ & $\mathrm{Q}(15)-\operatorname{In}(13)-\mathrm{Q}(19)$ & $114.36(5)$ \\
\hline $\mathrm{Q}(31)-\mathrm{M}(3)-\mathrm{Q}(28)$ & $111.13(6)$ & $\mathrm{Q}(27)-\operatorname{In}(14)-\mathrm{Q}(26)$ & $106.55(5)$ \\
\hline $\mathrm{Q}(31)-\mathrm{M}(3)-\mathrm{Q}(19)$ & $112.49(5)$ & $\mathrm{Q}(27)-\operatorname{In}(14)-\mathrm{Q}(11)$ & $108.80(5)$ \\
\hline $\mathrm{Q}(28)-\mathrm{M}(3)-\mathrm{Q}(19)$ & $106.67(5)$ & $\mathrm{Q}(26)-\operatorname{In}(14)-\mathrm{Q}(11)$ & $109.29(5)$ \\
\hline $\mathrm{Q}(31)-\mathrm{M}(3)-\mathrm{Q}(23)$ & $112.21(6)$ & $\mathrm{Q}(27)-\operatorname{In}(14)-\mathrm{Q}(23)$ & $109.93(5)$ \\
\hline $\mathrm{Q}(28)-\mathrm{M}(3)-\mathrm{Q}(23)$ & $106.75(5)$ & $\mathrm{Q}(26)-\operatorname{In}(14)-\mathrm{Q}(23)$ & $109.50(5)$ \\
\hline $\mathrm{Q}(19)-\mathrm{M}(3)-\mathrm{Q}(23)$ & $107.25(5)$ & $\mathrm{Q}(11)-\operatorname{In}(14)-\mathrm{Q}(23)$ & $112.60(5)$ \\
\hline $\mathrm{Q}(31)-\mathrm{M}(4)-\mathrm{Q}(7)$ & $111.14(5)$ & $\mathrm{Q}(30)-\operatorname{In}(15)-\mathrm{Q}(24)$ & $105.68(5)$ \\
\hline $\mathrm{Q}(31)-\mathrm{M}(4)-\mathrm{Q}(16)$ & $110.78(5)$ & $\mathrm{Q}(30)-\operatorname{In}(15)-\mathrm{Q}(16)$ & $107.79(5)$ \\
\hline $\mathrm{Q}(7)-\mathrm{M}(4)-\mathrm{Q}(16)$ & $108.20(5)$ & $\mathrm{Q}(24)-\operatorname{In}(15)-\mathrm{Q}(16)$ & $109.41(5)$ \\
\hline $\mathrm{Q}(31)-\mathrm{M}(4)-\mathrm{Q}(15)$ & $111.76(5)$ & $\mathrm{Q}(30)-\operatorname{In}(15)-\mathrm{Q}(28)$ & $110.28(5)$ \\
\hline $\mathrm{Q}(7)-\mathrm{M}(4)-\mathrm{Q}(15)$ & $107.87(5)$ & $\mathrm{Q}(24)-\operatorname{In}(15)-\mathrm{Q}(28)$ & $109.80(5)$ \\
\hline $\mathrm{Q}(16)-\mathrm{M}(4)-\mathrm{Q}(15)$ & $106.91(5)$ & $\mathrm{Q}(16)-\operatorname{In}(15)-\mathrm{Q}(28)$ & $113.56(5)$ \\
\hline $\mathrm{Cl}(1)-\operatorname{In}(1)-\mathrm{Q}(1)$ & $100.45(10)$ & $\mathrm{Q}(29)-\mathrm{In}(16)-\mathrm{Q}(27)$ & $106.12(5)$ \\
\hline $\mathrm{Cl}(1)-\operatorname{In}(1)-\mathrm{Q}(3)$ & $101.79(10)$ & $\mathrm{Q}(29)-\operatorname{In}(16)-\mathrm{Q}(28)$ & $106.72(5)$ \\
\hline $\mathrm{Q}(1)-\operatorname{In}(1)-\mathrm{Q}(3)$ & $115.40(6)$ & $\mathrm{Q}(27)-\operatorname{In}(16)-\mathrm{Q}(28)$ & $110.67(5)$ \\
\hline $\mathrm{Cl}(1)-\operatorname{In}(1)-\mathrm{Q}(2)$ & $101.81(10)$ & $\mathrm{Q}(29)-\operatorname{In}(16)-\mathrm{Q}(18)$ & $109.65(5)$ \\
\hline $\mathrm{Q}(1)-\operatorname{In}(1)-\mathrm{Q}(2)$ & $117.57(6)$ & $Q(27)-\operatorname{In}(16)-Q(18)$ & $108.88(5)$ \\
\hline $\mathrm{Q}(3)-\operatorname{In}(1)-\mathrm{Q}(2)$ & $115.71(5)$ & $\mathrm{Q}(28)-\operatorname{In}(16)-\mathrm{Q}(18)$ & $114.47(5)$ \\
\hline
\end{tabular}

*: $\mathrm{M}(1)=0.75 \mathrm{Cd}(1)+0.25 \mathrm{In}(1 \mathrm{~B}) ; \mathrm{M}(2)=0.75 \mathrm{Cd}(2)+0.25 \mathrm{In}(2 \mathrm{~B}) ; \mathrm{M}(3)=0.75 \mathrm{Cd}(3)+0.25 \operatorname{In}(3 \mathrm{~B}) ; \mathrm{M}(4)=$ $0.75 \mathrm{Cd}(4)+0.25 \operatorname{In}(4 \mathrm{~B})$

Table S8. Selected bond lengths $[\AA]$ and angles $\left[{ }^{\circ}\right]$ for T4-3.

\begin{tabular}{|c|c|c|c|}
\hline $\mathrm{M}(1)-\mathrm{Se}(4)$ & $2.6011(15)$ & $\operatorname{In}(3)-\operatorname{Se}(3) \# 2$ & $2.5430(15)$ \\
\hline $\mathrm{M}(1)-\mathrm{Se}(5)$ & $2.5976(14)$ & $\operatorname{In}(3)-\operatorname{Se}(5)$ & $2.6112(14)$ \\
\hline
\end{tabular}




\begin{tabular}{|c|c|c|c|}
\hline $\mathrm{M}(1)-\mathrm{Se}(6)$ & $2.6136(11)$ & $\operatorname{In}(3)-\operatorname{Se}(7)$ & $2.5456(15)$ \\
\hline $\mathrm{M}(1)-\mathrm{Se}(8) \# 1$ & $2.6116(14)$ & $\operatorname{In}(3)-\operatorname{Se}(8)$ & $2.6229(15)$ \\
\hline $\mathrm{M}(2)-\mathrm{Se}(6)$ & $2.617(2)$ & $\operatorname{In}(4)-\operatorname{Se}(5)$ & $2.6040(15)$ \\
\hline $\mathrm{M}(2)-\mathrm{Se}(10) \# 2$ & $2.6053(14)$ & $\operatorname{In}(4)-\operatorname{Se}(9)$ & $2.5500(16)$ \\
\hline $\mathrm{M}(2)-\mathrm{Se}(10) \# 1$ & $2.6053(14)$ & $\operatorname{In}(4)-\operatorname{Se}(10)$ & $2.6235(15)$ \\
\hline $\mathrm{M}(2)-\mathrm{Se}(10)$ & $2.6052(14)$ & $\operatorname{In}(4)-\operatorname{Se}(11)$ & $2.5456(16)$ \\
\hline $\operatorname{In}(1)-\operatorname{Se}(2)$ & $2.5608(13)$ & $\operatorname{In}(5)-\operatorname{Se}(8)$ & $2.6273(15)$ \\
\hline $\operatorname{In}(1)-\operatorname{Se}(2) \# 2$ & $2.5608(13)$ & $\operatorname{In}(5)-\operatorname{Se}(9) \# 2$ & $2.5506(16)$ \\
\hline $\operatorname{In}(1)-\operatorname{Se}(2) \# 1$ & $2.5608(13)$ & $\operatorname{In}(5)-\operatorname{Se}(10)$ & $2.6195(15)$ \\
\hline $\operatorname{In}(1)-\mathrm{Cl}(1)$ & $2.471(5)$ & $\operatorname{In}(5)-\operatorname{Se}(12)$ & $2.5378(15)$ \\
\hline $\operatorname{In}(2)-\operatorname{Se}(2)$ & $2.5429(15)$ & $\operatorname{In}(6)-\operatorname{Se}(7)$ & $2.5494(17)$ \\
\hline $\operatorname{In}(2)-\operatorname{Se}(3)$ & $2.5477(14)$ & $\operatorname{In}(6)-\operatorname{Se}(11)$ & $2.5673(17)$ \\
\hline $\operatorname{In}(2)-\operatorname{Se}(4)$ & $2.6184(14)$ & $\operatorname{In}(6)-\operatorname{Se}(12)$ & $2.5644(18)$ \\
\hline $\operatorname{In}(2)-\operatorname{Se}(4) \# 1$ & $2.6231(14)$ & $\operatorname{In}(6)-\mathrm{Cl}(2)$ & $2.478(4)$ \\
\hline $\operatorname{Se}(4)-M(1)-\operatorname{Se}(6)$ & $111.86(5)$ & $\operatorname{Se}(3) \# 2-\operatorname{In}(3)-\operatorname{Se}(5)$ & $106.52(5)$ \\
\hline $\operatorname{Se}(4)-\mathrm{M}(1)-\mathrm{Se}(8) \# 1$ & $106.56(5)$ & $\operatorname{Se}(3) \# 2-\operatorname{In}(3)-\operatorname{Se}(7)$ & $107.60(5)$ \\
\hline $\operatorname{Se}(5)-\mathrm{M}(1)-\mathrm{Se}(4)$ & $108.15(5)$ & $\operatorname{Se}(3) \# 2-\operatorname{In}(3)-\operatorname{Se}(8)$ & $112.52(5)$ \\
\hline $\operatorname{Se}(5)-M(1)-\operatorname{Se}(6)$ & $109.70(4)$ & $\operatorname{Se}(5)-\operatorname{In}(3)-\operatorname{Se}(8)$ & $113.64(5)$ \\
\hline $\operatorname{Se}(5)-M(1)-S e(8) \# 1$ & $108.15(5)$ & $\operatorname{Se}(7)-\operatorname{In}(3)-\operatorname{Se}(5)$ & $109.46(5)$ \\
\hline $\operatorname{Se}(8) \# 1-\mathrm{M}(1)-\operatorname{Se}(6)$ & $112.25(4)$ & $\operatorname{Se}(7)-\operatorname{In}(3)-\operatorname{Se}(8)$ & $106.95(5)$ \\
\hline $\operatorname{Se}(10) \# 1-\mathrm{M}(2)-\mathrm{Se}(6)$ & $111.32(4)$ & $\operatorname{Se}(5)-\operatorname{In}(4)-\operatorname{Se}(10)$ & $113.85(5)$ \\
\hline $\operatorname{Se}(10) \# 2-\mathrm{M}(2)-\mathrm{Se}(6)$ & $111.32(4)$ & $\operatorname{Se}(9)-\operatorname{In}(4)-\operatorname{Se}(5)$ & $108.16(5)$ \\
\hline $\operatorname{Se}(10)-\mathrm{M}(2)-\operatorname{Se}(6)$ & $111.31(4)$ & $\operatorname{Se}(9)-\operatorname{In}(4)-\operatorname{Se}(10)$ & $110.05(5)$ \\
\hline $\operatorname{Se}(10) \# 2-M(2)-\operatorname{Se}(10) \# 1$ & $107.57(4)$ & $\operatorname{Se}(11)-\operatorname{In}(4)-\operatorname{Se}(5)$ & $107.57(5)$ \\
\hline $\operatorname{Se}(10)-M(2)-\operatorname{Se}(10) \# 1$ & $107.57(4)$ & $\operatorname{Se}(11)-\operatorname{In}(4)-\operatorname{Se}(9)$ & $108.07(5)$ \\
\hline $\operatorname{Se}(10)-M(2)-\operatorname{Se}(10) \# 2$ & $107.57(4)$ & $\operatorname{Se}(11)-\operatorname{In}(4)-\operatorname{Se}(10)$ & $108.97(5)$ \\
\hline $\operatorname{Se}(2) \# 1-\operatorname{In}(1)-\operatorname{Se}(2) \# 2$ & $116.10(2)$ & $\operatorname{Se}(9) \# 2-\operatorname{In}(5)-\operatorname{Se}(8)$ & $107.66(5)$ \\
\hline $\operatorname{Se}(2)-\operatorname{In}(1)-\operatorname{Se}(2) \# 2$ & $116.10(2)$ & $\operatorname{Se}(9) \# 2-\operatorname{In}(5)-\operatorname{Se}(10)$ & $110.27(5)$ \\
\hline $\operatorname{Se}(2) \# 1-\operatorname{In}(1)-\operatorname{Se}(2)$ & $116.11(2)$ & $\operatorname{Se}(10)-\operatorname{In}(5)-\operatorname{Se}(8)$ & $116.57(5)$ \\
\hline $\mathrm{Cl}(1)-\mathrm{In}(1)-\mathrm{Se}(2) \# 2$ & $101.54(4)$ & $\operatorname{Se}(12)-\operatorname{In}(5)-\operatorname{Se}(8)$ & $108.99(5)$ \\
\hline $\mathrm{Cl}(1)-\operatorname{In}(1)-\mathrm{Se}(2)$ & $101.54(4)$ & $\operatorname{Se}(12)-\operatorname{In}(5)-\operatorname{Se}(9) \# 2$ & $107.10(5)$ \\
\hline $\mathrm{Cl}(1)-\operatorname{In}(1)-\mathrm{Se}(2) \# 1$ & $101.54(4)$ & $\operatorname{Se}(12)-\operatorname{In}(5)-\operatorname{Se}(10)$ & $105.86(5)$ \\
\hline $\operatorname{Se}(2)-\operatorname{In}(2)-\operatorname{Se}(3)$ & $107.21(5)$ & $\operatorname{Se}(7)-\operatorname{In}(6)-\operatorname{Se}(11)$ & $116.90(6)$ \\
\hline $\operatorname{Se}(2)-\operatorname{In}(2)-\operatorname{Se}(4)$ & $105.08(5)$ & $\operatorname{Se}(7)-\operatorname{In}(6)-\operatorname{Se}(12)$ & $117.74(6)$ \\
\hline $\operatorname{Se}(2)-\operatorname{In}(2)-\operatorname{Se}(4) \# 1$ & $111.12(5)$ & $\operatorname{Se}(12)-\operatorname{In}(6)-\operatorname{Se}(11)$ & $114.16(6)$ \\
\hline $\operatorname{Se}(3)-\operatorname{In}(2)-\operatorname{Se}(4)$ & $111.42(5)$ & $\mathrm{Cl}(2)-\operatorname{In}(6)-\operatorname{Se}(7)$ & $101.21(11)$ \\
\hline $\operatorname{Se}(3)-\operatorname{In}(2)-\operatorname{Se}(4) \# 1$ & $106.76(4)$ & $\mathrm{Cl}(2)-\operatorname{In}(6)-\mathrm{Se}(11)$ & $101.98(11)$ \\
\hline $\operatorname{Se}(4)-\operatorname{In}(2)-\operatorname{Se}(4) \# 1$ & $115.05(5)$ & $\mathrm{Cl}(2)-\operatorname{In}(6)-\mathrm{Se}(12)$ & $100.66(11)$ \\
\hline
\end{tabular}


(a)

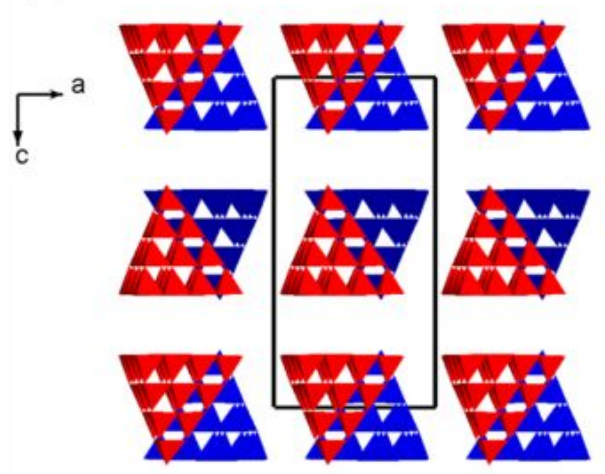

(b)

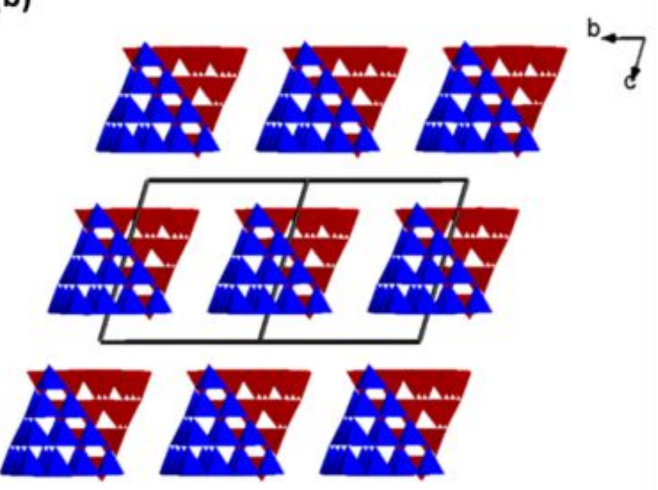

Figure S1. (a) Arrangement way of anions in T4-1 and T4-3; (b) arrangement way of anions in T4-2.

\section{More characterizations}

1) PXRD and TGA curves
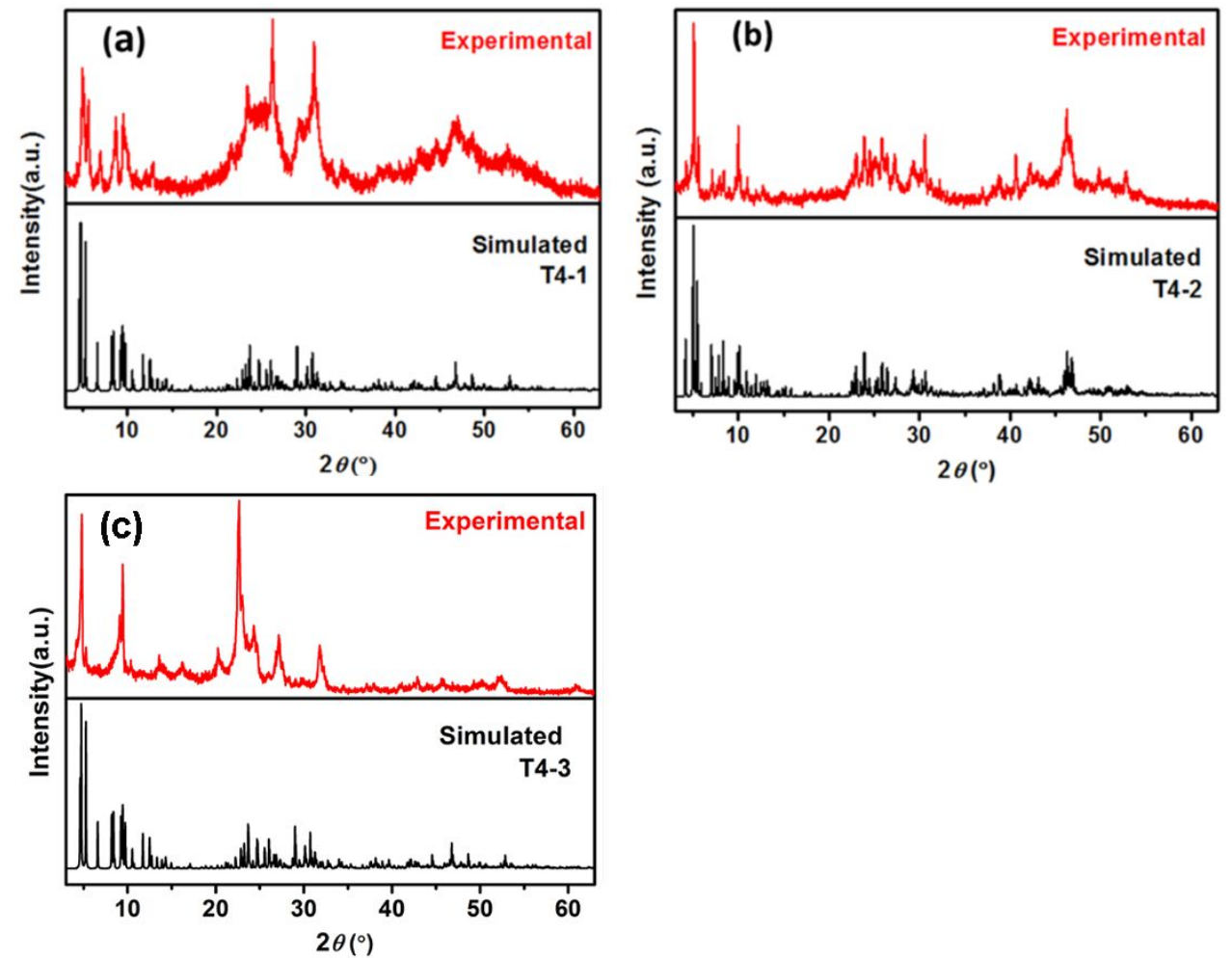

Figure S2. PXRD patterns of title compounds and that simulated from single crystal X-ray structures. 

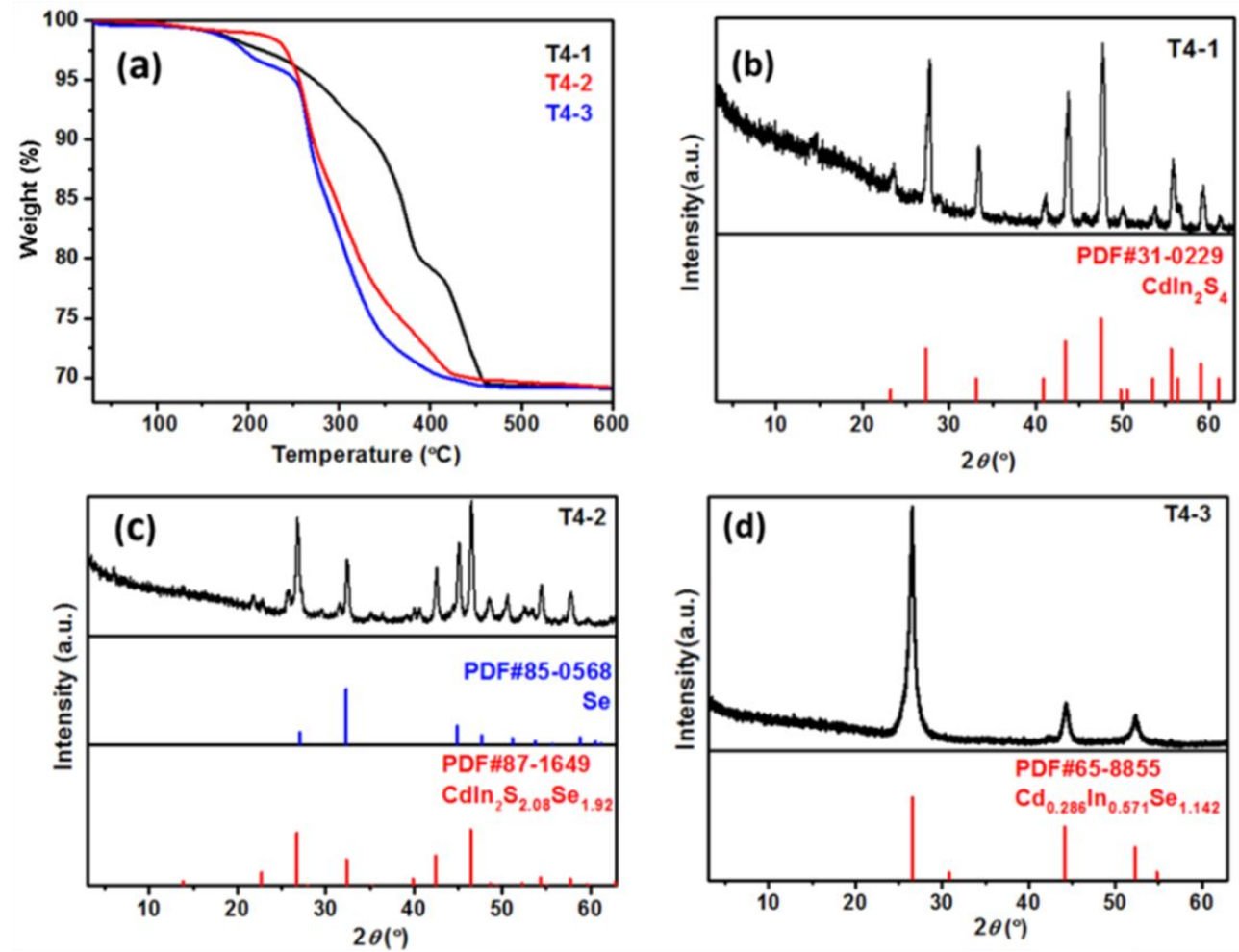

Figure S3. (a) TGA curves of title compounds; (b)-(d) PXRD patterns of the TGA residues of T4-1, T4-2 and T4-3 compared with that of corresponding phases based on JCPDS cards.

2) EDS and ICP data of T4 clusters
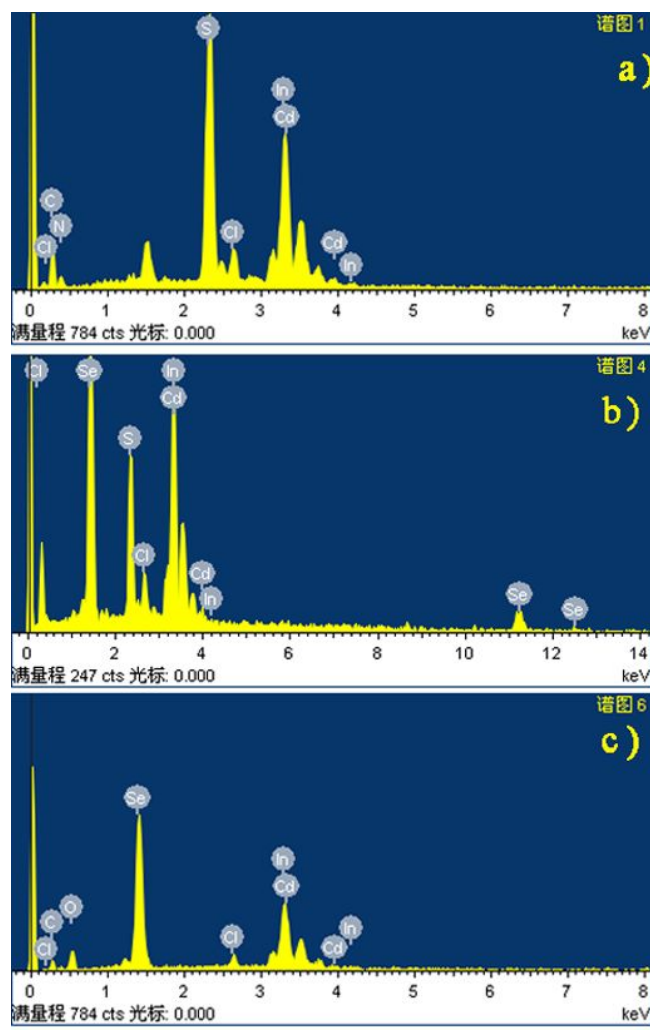

Figure S4. EDS spectra of T4-1 (a), T4-2 (b) and T4-3 (c). The contents of chemical elements in T4 clusters are $\mathrm{CdIn}_{4.79} \mathrm{~S}_{7.23} \mathrm{Cl}_{1.30}$ for T4-1, CdIn ${ }_{5.40} \mathrm{~S}_{4.72} \mathrm{Se}_{6.02} \mathrm{Cl}_{1.35}$ for T4-2 and $\mathrm{CdIn}_{5.03} \mathrm{Se}_{10.20} \mathrm{Cl}_{1.21}$ for T4-3, respectively. 
Table S9. The contents of cadmium and indium in T4 clusters.

\begin{tabular}{cccc}
\hline Compounds & T4-1 & T4-2 & T4-3 \\
\hline Cd/In wt \% from the ICP data & $7.36 / 39.71$ & $5.95 / 35.45$ & $5.36 / 30.82$ \\
Cd/In wt \% from the Formula & $7.02 / 40.63$ & $5.97 / 34.56$ & $5.26 / 30.43$ \\
\hline
\end{tabular}

3) ESI-MS of T4-2 and T4-3

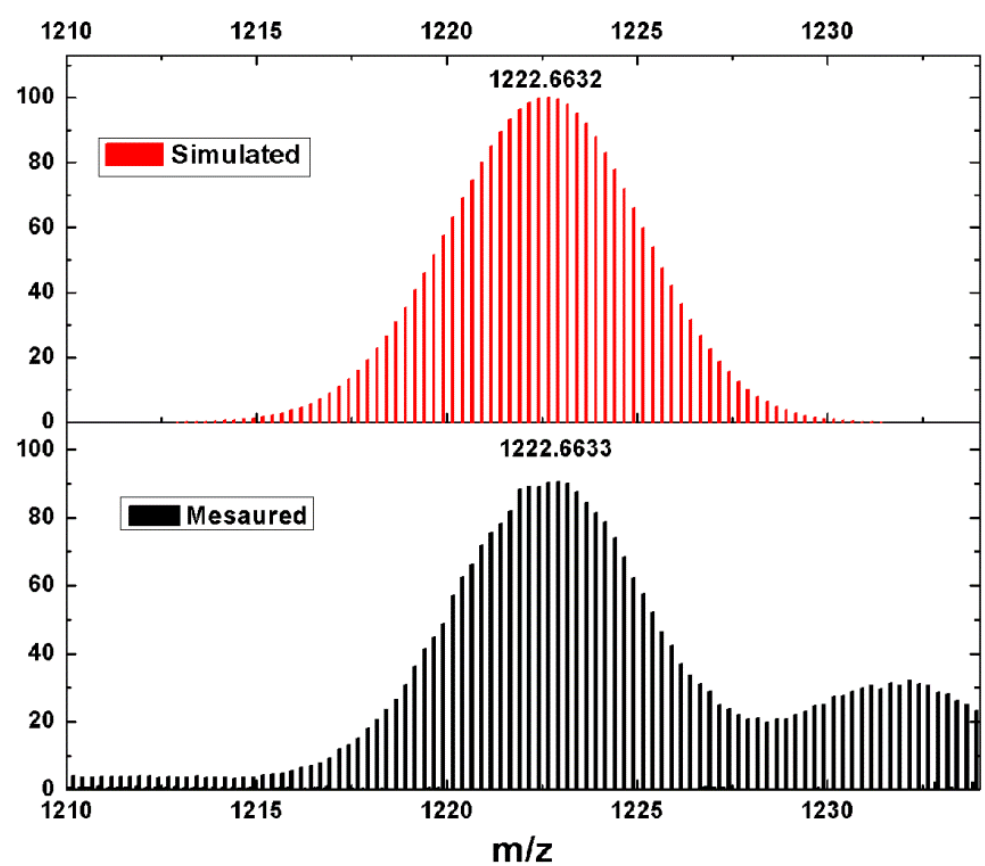

Figure S5. The measured mass spectrum (bottom) of the $\left[(\mathrm{BMMim})\left(\mathrm{Cd}_{3} \operatorname{In}_{17} \mathrm{Se}_{31}\right)\right]^{4-}$ clusters is comparable with that simulated from isotopic patterns (top).

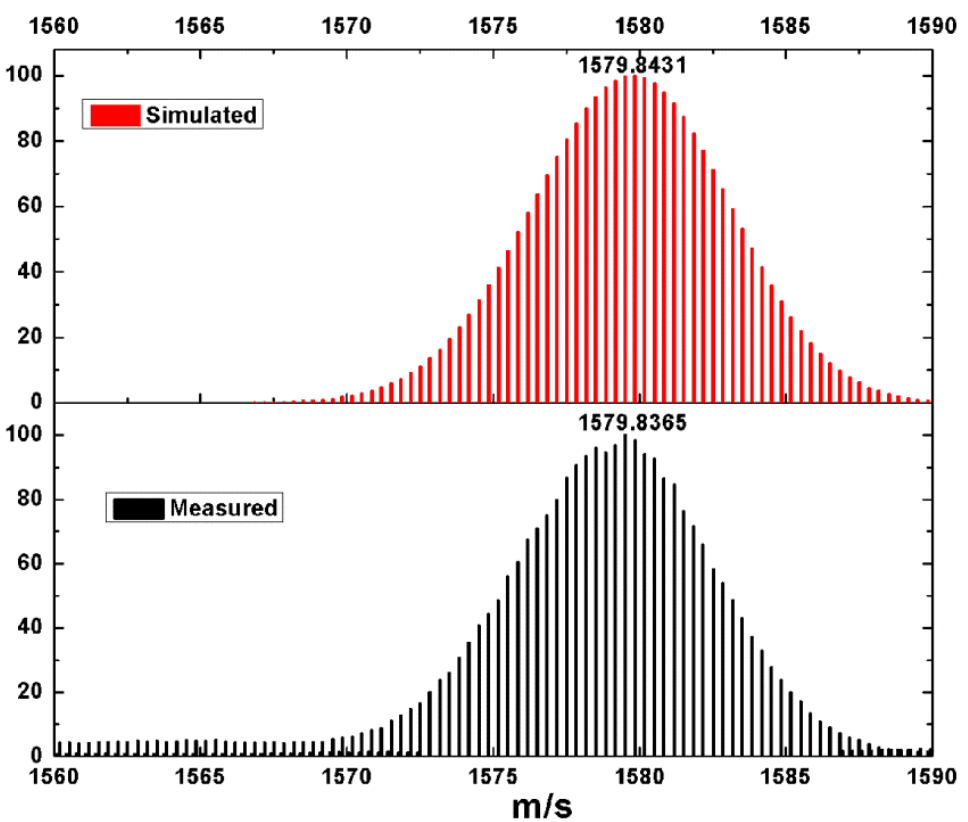

Figure S6. The measured mass spectrum (bottom) of the $\left[\mathrm{H}_{2}\left(\mathrm{Cd}_{3} \mathrm{In}_{17} \mathrm{Se}_{31}\right)\right]^{3-}$ clusters is comparable with that simulated from isotopic patterns (top). 


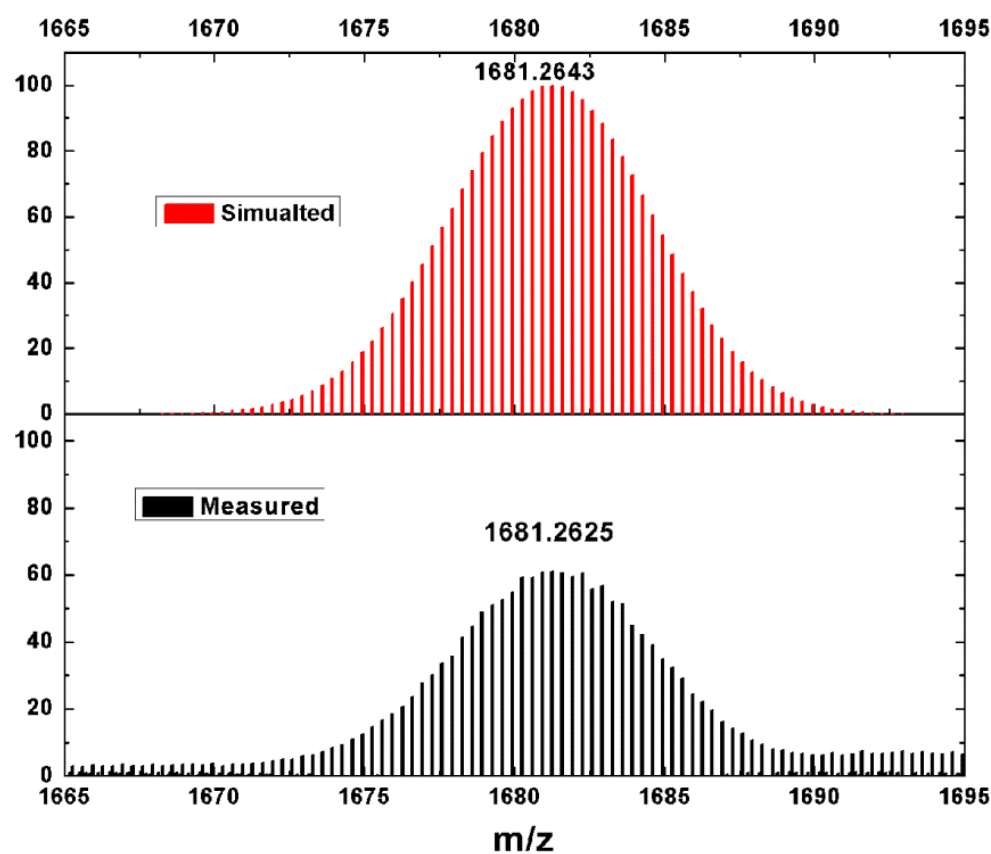

Figure S7. The measured mass spectrum (bottom) of the $\left[(\mathrm{BMMim})_{2}\left(\mathrm{Cd}_{3} \operatorname{In}_{17} \mathrm{Se}_{31}\right)\right]^{3-}$ clusters is comparable with that simulated from isotopic patterns (top).
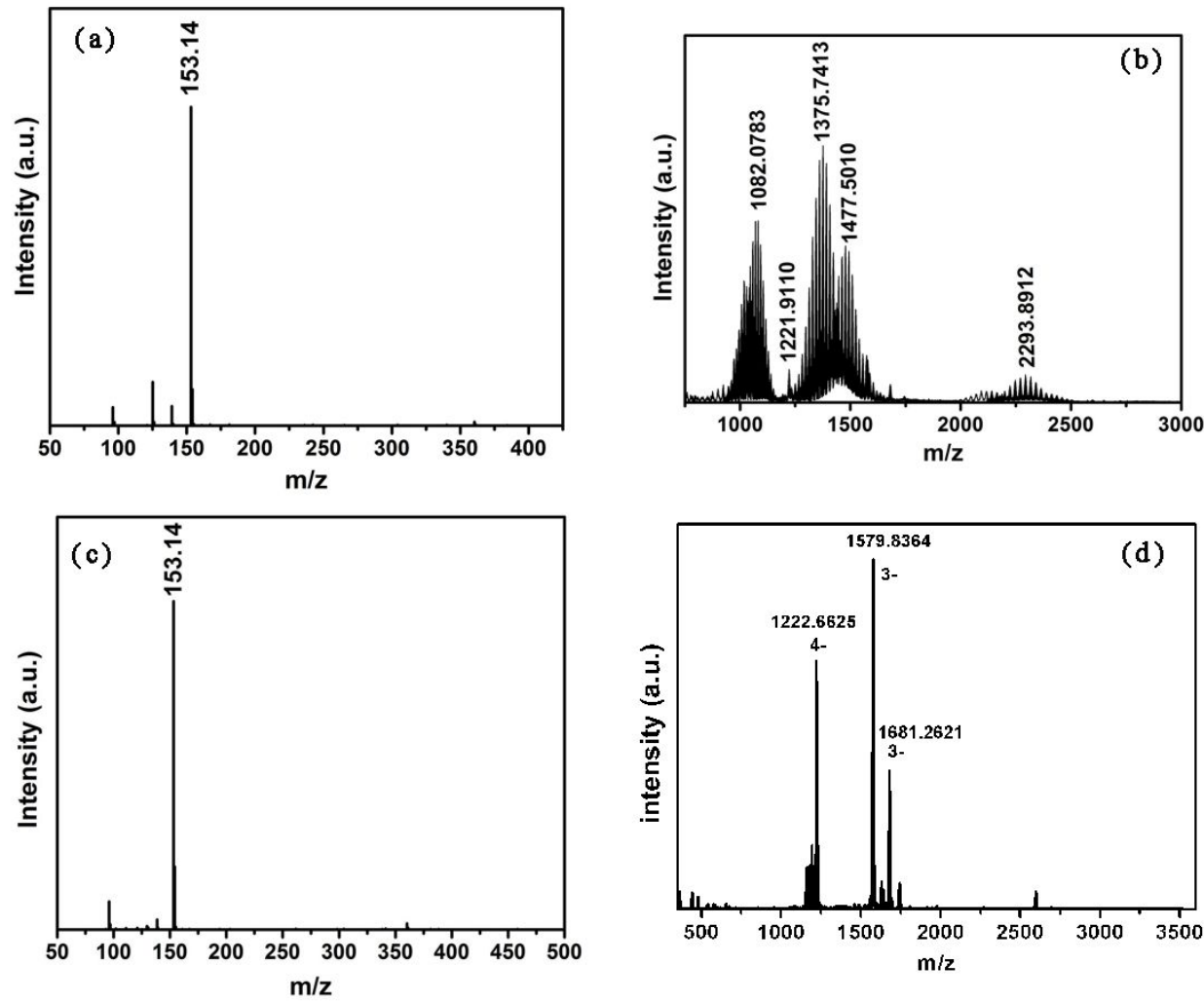

Figure S8. (a) Positive mode ESI-MS spectrum of T4-2 solution; (b) negative mode ESI-MS spectrum of T4-2 solution; (c) positive mode ESI-MS spectrum of T4-3 solution; (d) negative mode ESI-MS spectrum of T4-3 solution kept in the ambient conditions for 7 days; $10 \mathrm{mg}$ samples were dispersed in $4 \mathrm{~mL}$ DMSO, which were diluted by methanol and immediately injected into the MS instrument. 
3) Stability after dispersed T4-2 and T4-3 clusters in DMSO solution

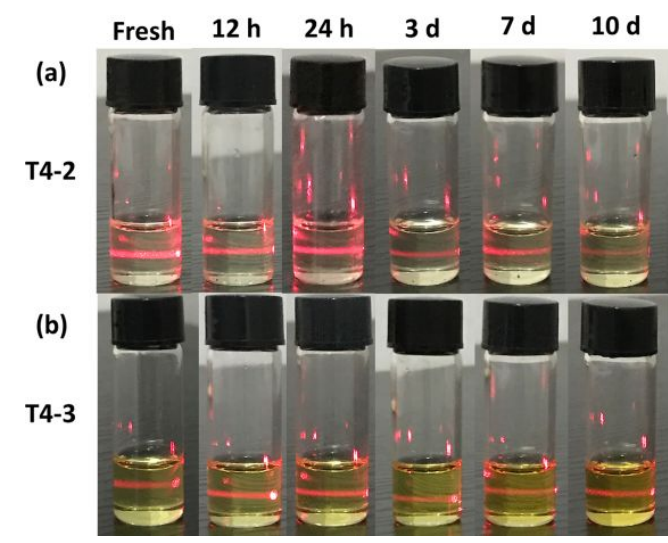

Figure S9. Photos of the dispersed T4-2 (a) and T4-3 (b) clusters in DMSO solution $(20 \mathrm{mg} / \mathrm{mL})$ under ambient conditions for different times.

4) Mott-Schottky plots
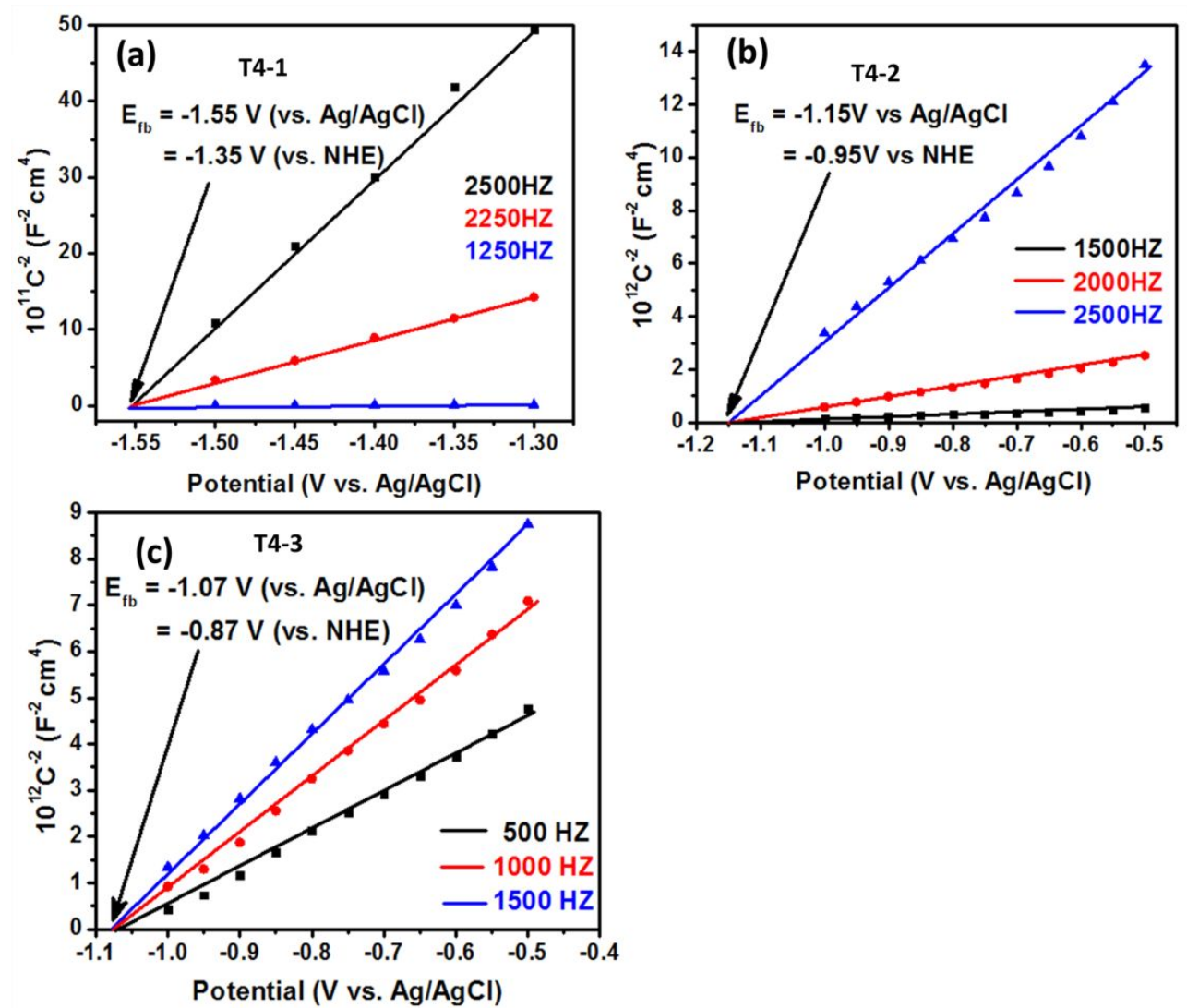

Figure S10. Mott-schottky plots of the title compounds at different frequency. 
Table S10 Calculated results for the band positions of T4-1, T4-2 and T4-3.

\begin{tabular}{|c|c|c|c|c|c|c|}
\hline $\begin{array}{l}\text { Sample } \\
\text { ID }\end{array}$ & $\begin{array}{c}\mathrm{E}_{\mathrm{fb}} \\
\mathrm{V}, \mathrm{vs} . \\
\mathrm{Ag} / \mathrm{AgCl} \\
\end{array}$ & $\begin{array}{c}\mathrm{E}_{\mathrm{fb}} \\
\mathrm{V}, \mathrm{vs} . \\
\mathrm{NHE}\end{array}$ & $\begin{array}{c}\mathrm{E}_{\mathrm{CB}} \\
\mathrm{V}, \mathrm{vs} . \\
\mathrm{NHE} \\
\end{array}$ & $\begin{array}{c}\mathrm{E}_{\mathrm{VB}} \\
\mathrm{V}, \mathrm{vs} . \\
\text { NHE }\end{array}$ & $\begin{array}{c}\mathrm{E}_{\mathrm{CB}} \\
\text { eV, vs. Vacuum } \\
\text { Level } \\
\end{array}$ & $\begin{array}{c}\mathrm{E}_{\mathrm{VB}} \\
\text { eV, vs. Vacuum } \\
\text { Level } \\
\end{array}$ \\
\hline T4-1 & -1.55 & -1.35 & -1.45 & 1.80 & -2.99 & -6.24 \\
\hline T4-2 & -1.15 & -0.95 & -1.05 & 1.71 & -3.39 & -6.15 \\
\hline T4-3 & -1.07 & -0.87 & -0.97 & 1.63 & -3.47 & -6.07 \\
\hline
\end{tabular}

With $\mathrm{Ag} / \mathrm{AgCl}$ electrode as the reference electrode, relative to the NHE potential of $0.20 \mathrm{~V},{ }^{10}$ the formula for calculation level:

$\mathrm{E}_{\mathrm{fb}}(\mathrm{V}, \mathrm{vs} . \mathrm{NHE})=\mathrm{E}_{\mathrm{fb}}(\mathrm{V}, \mathrm{vs} . \mathrm{Ag} / \mathrm{AgCl})+0.20 \mathrm{~V}$

$\mathrm{E}_{\mathrm{CB}}(\mathrm{V}, \mathrm{vs} . \mathrm{NHE})=\mathrm{E}_{\mathrm{fb}}(\mathrm{V}, \mathrm{vs}$. NHE) $-0.1 \mathrm{~V}$

$\mathrm{E}_{\mathrm{CB}}\left(\mathrm{eV}\right.$, vs. Vacuum Level) $=-\mathrm{E}_{\mathrm{CB}}(\mathrm{V}$, vs. NHE) - 4.44 (at 298K)

$\mathrm{E}_{\mathrm{VB}}(\mathrm{eV}$, vs. Vacuum Level $)=\mathrm{E}_{\mathrm{CB}}\left(\mathrm{eV}\right.$, vs. Vacuum Level)] $-\mathrm{E}_{\mathrm{BG}}$

$E_{V B}\left(V\right.$, vs. NHE) $=-E_{V B}(e V$, vs. Vacuum Level) -4.44 (at 298K)

\section{For T4-1}

$\mathrm{E}_{\mathrm{fb}}(\mathrm{V}, \mathrm{vs} . \mathrm{NHE})=\mathrm{E}_{\mathrm{fb}}(\mathrm{V}$, vs. $\mathrm{Ag} / \mathrm{AgCl})+0.20 \mathrm{~V}=-1.55 \mathrm{~V}+0.20 \mathrm{~V}=-1.35 \mathrm{~V}$

$\mathrm{E}_{\mathrm{CB}}(\mathrm{V}, \mathrm{vs}$. NHE $)=\mathrm{E}_{\mathrm{fb}}(\mathrm{V}, \mathrm{vs}$. NHE) $-0.1 \mathrm{~V}=-1.45 \mathrm{~V}$

$\mathrm{E}_{\mathrm{CB}}(\mathrm{eV}$, vs. Vacuum Level $)=-\mathrm{E}_{\mathrm{CB}}(\mathrm{V}$, vs. NHE) - $4.44($ at $298 \mathrm{~K})=-2.99 \mathrm{eV}$

$\mathrm{E}_{\mathrm{VB}}\left(\mathrm{eV}\right.$, vs. Vacuum Level) $=\mathrm{E}_{\mathrm{CB}}\left(\mathrm{eV}\right.$, vs. Vacuum Level)] $-\mathrm{E}_{\mathrm{BG}}=-6.24 \mathrm{eV}$

$\mathrm{E}_{\mathrm{VB}}(\mathrm{V}$, vs. NHE $)=-\mathrm{E}_{\mathrm{VB}}(\mathrm{eV}$, vs. Vacuum Level $)-4.44($ at $298 \mathrm{~K})=1.80 \mathrm{~V}$

\section{For T4-2,}

$\mathrm{E}_{\mathrm{fb}}(\mathrm{V}$, vs. $\mathrm{NHE})=\mathrm{E}_{\mathrm{fb}}(\mathrm{V}$, vs. $\mathrm{Ag} / \mathrm{AgCl})+0.20 \mathrm{~V}=-1.15 \mathrm{~V}+0.20 \mathrm{~V}=-0.95 \mathrm{~V}$

$\mathrm{E}_{\mathrm{CB}}(\mathrm{V}, \mathrm{vs}$. NHE $)=\mathrm{E}_{\mathrm{fb}}(\mathrm{V}$, vs. NHE) $-0.1 \mathrm{~V}=-1.05 \mathrm{~V}$

$\mathrm{E}_{\mathrm{CB}}\left(\mathrm{eV}\right.$, vs. Vacuum Level) $=-\mathrm{E}_{\mathrm{CB}}(\mathrm{V}$, vs. NHE) - $4.44($ at $298 \mathrm{~K})=-3.39 \mathrm{eV}$

$\mathrm{E}_{\mathrm{VB}}\left(\mathrm{eV}\right.$, vs. Vacuum Level) $=\mathrm{E}_{\mathrm{CB}}\left(\mathrm{eV}\right.$, vs. Vacuum Level)] $-\mathrm{E}_{\mathrm{BG}}=-6.15 \mathrm{eV}$

$\mathrm{E}_{\mathrm{VB}}(\mathrm{V}, \mathrm{vs} . \mathrm{NHE})=-\mathrm{E}_{\mathrm{VB}}(\mathrm{eV}$, vs. Vacuum Level) $-4.44($ at $298 \mathrm{~K})=1.71 \mathrm{~V}$

\section{For T4-3,}

$\mathrm{E}_{\mathrm{fb}}(\mathrm{V}, \mathrm{vs} . \mathrm{NHE})=\mathrm{E}_{\mathrm{fb}}(\mathrm{V}, \mathrm{vs} . \mathrm{Ag} / \mathrm{AgCl})+0.20 \mathrm{~V}=-1.07 \mathrm{~V}+0.20 \mathrm{~V}=-0.87 \mathrm{~V}$

$\mathrm{E}_{\mathrm{CB}}(\mathrm{V}$, vs. NHE $)=\mathrm{E}_{\mathrm{fb}}(\mathrm{V}$, vs. NHE) $-0.1 \mathrm{~V}=-0.97 \mathrm{~V}$

$\mathrm{E}_{\mathrm{CB}}(\mathrm{eV}$, vs. Vacuum Level $)=-\mathrm{E}_{\mathrm{CB}}(\mathrm{V}$, vs. NHE) - $4.44($ at $298 \mathrm{~K})=-3.47 \mathrm{eV}$

$\mathrm{E}_{\mathrm{VB}}(\mathrm{eV}$, vs. Vacuum Level $)=\mathrm{E}_{\mathrm{CB}}(\mathrm{eV}$, vs. Vacuum Level $\left.)\right]-\mathrm{E}_{\mathrm{BG}}=-6.07 \mathrm{eV}$

$\mathrm{E}_{\mathrm{VB}}\left(\mathrm{V}\right.$, vs. NHE) $=-\mathrm{E}_{\mathrm{VB}}(\mathrm{eV}$, vs. Vacuum Level) $-4.44($ at $298 \mathrm{~K})=1.63 \mathrm{~V}$ 


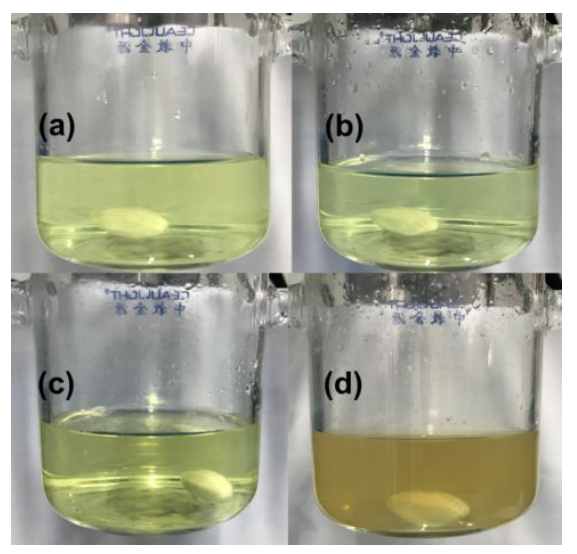

Figure S11. Photos of T4-3 solution before (a) and after (b) photocatalytic reaction without cocatalyst Pt; photos of T4-3 solution before (c) and after (d) photocatalytic reaction with cocatalyst Pt.
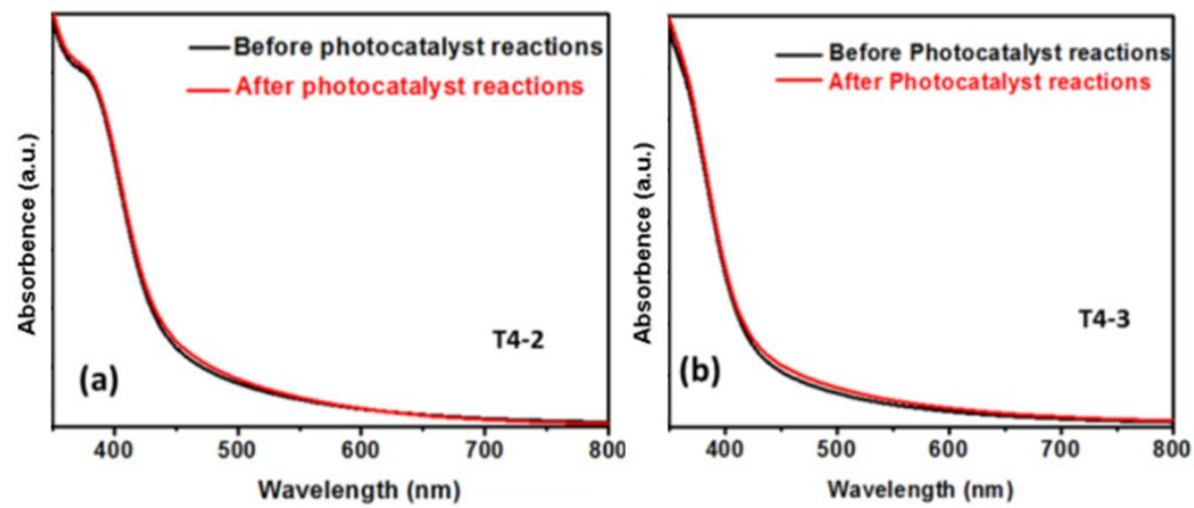

Figure S12. Liquid state UV-Vis absorption spectra for T4-2 and T4-3 before and after photocatalytic reaction.
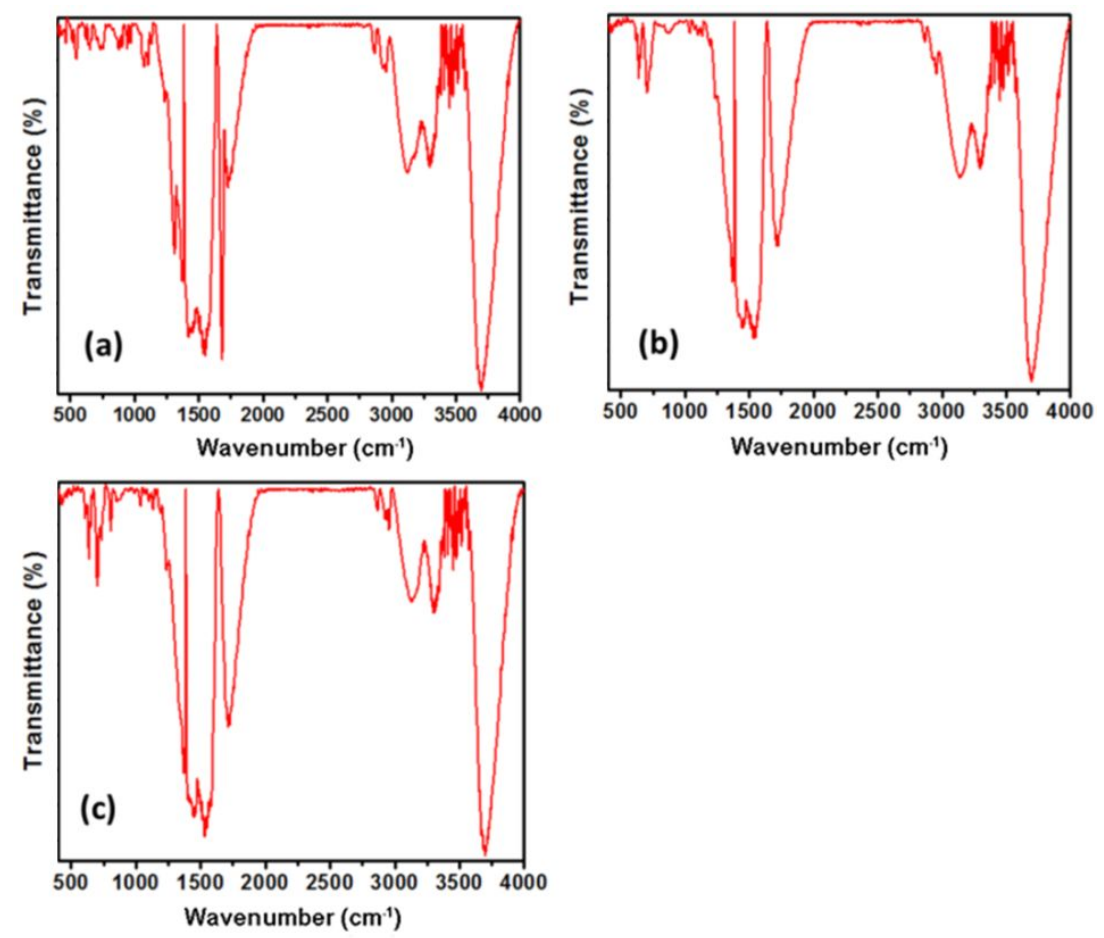

Figure S13. FT-IR spectra of T4-1 (a), T4-2 (b), and T4-3 (c). 


\section{References:}

(1) Sheldrick, G. M., Crystal structure refinement with SHELXL. Acta Crystallogr. 2015, C71, 3-8.

(2) Wendlandt, W. W.; Hecht, H. G., Reflectance spectroscopy, Interscience Publishers. New York, 1966.

(3) Zheng, N. F.; Bu, X. H.; Vu, H.; Feng, P. Y., Open-framework chalcogenides as visible-light photocatalysts for hydrogen generation from water. Angew. Chem. Int. Ed. 2005, 44, 5299-5303.

(4) Zhang, Z. Y.; Zhang, J.; Wu, T.; Bu, X. H.; Feng, P. Y., Three-dimensional open framework built from Cu-S icosahedral clusters and its photocatalytic property. J. Am. Chem. Soc. 2008, 130, 15238-15239.

(5) Wang, L.; Wu, T.; Zuo, F.; Zhao, X.; Bu, X. H.; Wu, J. Z.; Feng, P. Y., Assembly of supertetrahedral $T_{5}$ copper-indium sulfide clusters into a super-supertetrahedron of infinite order. J. Am. Chem. Soc. 2010, 132, 3283-3285.

(6) Lin, Q.; Bu, X.; Mao, C.; Zhao, X.; Sasan, K.; Feng, P., Mimicking high-silica zeolites: Highly stable germanium- and tin-rich zeolite-type chalcogenides. J. Am. Chem. Soc. 2015, 137, 6184-6187.

(7) Yue, C. Y.; Lei, X. W.; Feng, L. J.; Wang, C.; Gong, Y. P.; Liu, X. Y., [ $\left[\mathrm{Mn}_{2} \mathrm{Ga}_{4} \mathrm{Sn}_{4} \mathrm{~S}_{20}\right]^{8-} \mathrm{T} 3$ supertetrahedral nanocluster directed by a series of transition metal complexes. Dalton Trans. 2015, 44, 2416-2424.

(8) Du, C. F.; Li, J. R.; Zhang, B.; Shen, N. N.; Huang, X. Y., From T2,2@bmmim to alkali@T2,2@bmmim ivory ball-like clusters: Ionothermal syntheses, precise doping, and photocatalytic properties. Inorg. Chem. 2015, 54, 5874-5878.

(9) Shen, N. N.; Hu, B.; Cheng, C. C.; Zou, G. D.; Hu, Q. Q.; Du, C. F.; Li, J. R.; Huang, X. Y., Discrete supertetrahedral $\mathrm{T} 3 \mathrm{InQ}$ clusters $(\mathrm{Q}=\mathrm{S}, \mathrm{S} / \mathrm{Se}, \mathrm{Se}, \mathrm{Se} / \mathrm{Te})$ : Ionothermal syntheses and tunable optical and photodegradation properties. Cryst. Growth Des. 2018, 18, 962-968.

(10) Liu, H.; Xu, C.; Li, D.; Jiang, H. L., Photocatalytic hydrogen production coupled with selective benzylamine oxidation over MOF composites. Angew. Chem., Int. Ed. 2018, 57, 5379-5383. 